\title{
Relativistic corrections to nonrelativistic effective field theories
}

\author{
Mohammad Hossein Namjoo, ${ }^{*}$ Alan H. Guth, ${ }^{\dagger}$ and David I. Kaiser \\ Department of Physics, Massachusetts Institute of Technology, Cambridge, Massachusetts 02139, USA
}

(Received 13 June 2018; published 20 July 2018)

\begin{abstract}
In this paper we develop a formalism for studying the nonrelativistic limit of relativistic field theories in a systematic way. By introducing a simple, nonlocal field redefinition, we transform a given relativistic theory, describing a real, self-interacting scalar field, into an equivalent theory, describing a complex scalar field that encodes at each time both the original field and its conjugate momentum. Our low-energy effective theory incorporates relativistic corrections to the kinetic energy as well as the backreaction of fast-oscillating terms on the behavior of the dominant, slowly varying component of the field. Possible applications of our new approach include axion dark matter, though the methods developed here should be applicable to the low-energy limits of other field theories as well.
\end{abstract}

DOI: 10.1103/PhysRevD.98.016011

\section{INTRODUCTION}

Understanding the nature of dark matter remains a major challenge at the intersections of astrophysics, cosmology, and particle physics. Multiple lines of observational evidence indicate that dark matter should be plentiful throughout the universe, contributing roughly five times more to the energy density of the universe than ordinary (baryonic) matter, and that the dark matter should be cold and collisionless [1]. From the standpoint of particle theory, the puzzle of dark matter includes at least two components: identifying a plausible dark-matter candidate within realistic models of particle physics, and developing an accurate, theoretical description that is suitable for low-energy phenomena associated with cold matter. For the latter goal, it is important to develop a means of characterizing the nonrelativistic limit of relativistic quantum field theories in a systematic way.

In this paper we develop an effective field theory approach for the nonrelativistic limit of relativistic field theories. We focus on the nonrelativistic behavior of scalar fields in Minkowski spacetime, incorporating systematic relativistic corrections as well as corrections from the fields' (weakly coupled) self-interactions. We have in mind applications to axion dark matter [2-5], though the methods developed here should be applicable to the low-energy limits of other field theories, such as QED, QCD [6-8], and

\footnotetext{
*namjoo@mit.edu

† guth@ctp.mit.edu

dikaiser@mit.edu
}

Published by the American Physical Society under the terms of the Creative Commons Attribution 4.0 International license. Further distribution of this work must maintain attribution to the author(s) and the published article's title, journal citation, and DOI. Funded by SCOAP. condensed-matter systems, and to specific phenomena such as "oscillons" [9-11] and "superradiance" [12,13].

Axions are an attractive candidate for dark matter. The hypothetical particles were originally introduced to address the strong $C P$ problem in QCD [14-16], but their expected mass and self-coupling make them well-suited for cold dark matter as well. In particular, axions, with $m \sim 10^{-4}-10^{-5} \mathrm{eV}$, are expected to be produced early in cosmic history, around the time of the QCD phase transition. At that time the typical wavenumber was $k \sim H_{\mathrm{QCD}} \sim 10^{-11} \mathrm{eV}$ [17], where $H_{\mathrm{QCD}} \sim T_{\mathrm{QCD}}^{2} / M_{\mathrm{pl}}$ is the Hubble scale at the time of the QCD transition, $T_{\mathrm{QCD}} \sim$ $0.1 \mathrm{GeV}$ is the temperature at which the transition occurs, and $M_{\mathrm{pl}} \equiv 1 / \sqrt{8 \pi G} \sim 10^{18} \mathrm{GeV}$ is the reduced Planck mass. Axions are expected to become accelerated gravitationally during large-scale structure formation, up to speeds $v / c \sim \mathcal{O}\left(10^{-3}\right)$ within galactic halos [17]. Hence even at late times they should remain squarely within the nonrelativistic limit, albeit in a regime in which relativistic corrections may be competitive with other nontrivial corrections, such as from higher-order interaction terms.

In the nonrelativistic limit, fluctuations that oscillate rapidly on timescales $m^{-1}$ (where $m$ is the mass of the scalar field) may be expected to average to zero over timescales $\Delta t \gg m^{-1}$. However, nonlinear self-couplings can induce a backreaction of the fast-oscillating terms on the dominant, slowly varying component of the nonrelativistic field, affecting its behavior. Such coupling of fastand slow-oscillating terms produces measurable effects in many physical systems, such as neuronal processes related to memory formation $[18,19]$. We develop an iterative, perturbative procedure to incorporate this backreaction.

Our approach complements other recent work, such as Refs. [11,17,20]. In particular, the authors of Ref. [20] 
develop a nonrelativistic effective field theory for describing axions by calculating scattering amplitudes for $n \rightarrow n$ body scattering in the full, relativistic theory, and then taking the low-energy limit of those amplitudes to match coefficients in a series expansion of the effective potential. In Ref. [11], the authors developed an effective field theory by using diagrammatic techniques to integrate out the highmomentum modes. In this paper, we develop an effective description for the nonrelativistic limit with no need to calculate scattering amplitudes in the corresponding relativistic theory. Our method yields the same coefficients for the leading higher-order interaction terms in the low-energy limit as those found in Refs. [11,20], while also incorporating systematic relativistic corrections which do not appear in the previous analysis.

In Sec. II we introduce a convenient field redefinition to relate the real-valued scalar field described by the relativistic theory to a complex scalar field more appropriate to the nonrelativistic limit. In particular, we introduce a nonlocal field redefinition rather than the local redefinition that one typically finds in the literature. Although we expect the resulting descriptions of the low-energy limit to be equivalent in either redefinition, we find that the nonlocal redefinition considerably simplifies the derivation. In Sec. III we develop an effective field theory for the nonrelativistic field, which incorporates contributions from fast-oscillating terms on the evolution of the principal, slowly varying portion of the field. Concluding remarks follow in Sec. IV. In Appendix A we demonstrate that our transformation from the real-valued relativistic field $\phi(t, \mathbf{x})$ to the complex-valued nonrelativistic field $\psi(t, \mathbf{x})$ can be constructed as a canonical transformation. In Appendix B we demonstrate that the low-energy effective field theory based on our nonlocal field redefinition explicitly matches what one would calculate with the local field redefinition in two regimes of interest. In Appendix $\mathrm{C}$ we compare our results with the recent calculation in Ref. [11] and demonstrate that the two results are equivalent, related to each other by a field redefinition.

\section{FIELD REDEFINITION FOR A NONRELATIVISTIC FORMULATION}

In this section we begin with the Lagrangian for a relativistic field theory that describes a scalar field in Minkowski spacetime, and introduce a suitable field redefinition with which we may consider the nonrelativistic limit systematically. Our goal is to obtain an expression for the Lagrangian that yields the Schrödinger equation as the effective equation of motion for the redefined field in the extreme nonrelativistic limit.

We consider a relativistic scalar field of mass $m$ with a $\lambda \phi^{4}$ interaction, described by the Lagrangian density

$$
\mathcal{L}=-\frac{1}{2} \eta^{\mu \nu} \partial_{\mu} \phi \partial_{\nu} \phi-\frac{1}{2} m^{2} \phi^{2}-\frac{1}{4 !} \lambda \phi^{4},
$$

where $\phi$ is a real-valued scalar field, and we take the Minkowski metric to be $\eta_{\mu \nu}=\operatorname{diag}[-1,1,1,1]$. The canonical momentum field is $\pi=\dot{\phi}$, where an overdot denotes a derivative with respect to time, and the Hamiltonian is given by

$$
\begin{aligned}
& H=\int d^{3} x \mathcal{H}(\mathbf{x}), \\
& \mathcal{H}=\frac{1}{2} \pi^{2}+\frac{1}{2}(\mathbf{\nabla} \phi)^{2}+\frac{1}{2} m^{2} \phi^{2}+\frac{1}{4 !} \lambda \phi^{4} .
\end{aligned}
$$

The equations of motion take the form

$$
\begin{aligned}
& \dot{\phi}=\frac{\delta H}{\delta \pi}=\pi, \\
& \dot{\pi}=-\frac{\delta H}{\delta \phi}=\left(\nabla^{2}-m^{2}\right) \phi-\frac{1}{3 !} \lambda \phi^{3} .
\end{aligned}
$$

In contrast, the standard Lagrangian for a free, nonrelativistic field may be written

$$
\mathcal{L}=\frac{i}{2}\left(\dot{\psi} \psi^{*}-\psi \dot{\psi}^{*}\right)-\frac{1}{2 m} \nabla \psi \nabla \psi^{*},
$$

where $\psi$ is a complex scalar field, and overdots again denote derivatives with respect to time. The kinetic term is normalized so that the field $\psi$ obeys the standard Poisson bracket relations with $\psi^{*}$, so that when quantized the two fields obey the standard commutation relations, as given below in Eq. (10) and discussed in Eqs. (14)-(20). Whereas the Lagrangian in Eq. (1) yields a second-order equation of motion for $\phi$, the Lagrangian in Eq. (4) yields first order equations of motion for the real and imaginary parts of $\psi$. The Lagrangian in Eq. (4) has a global $U(1)$ symmetry; the associated conserved charge is simply the number of particles,

$$
N=\int d^{3} x|\psi|^{2}
$$

confirming the usual expectation that particle number should be conserved in a nonrelativistic theory, appropriate for energy scales $E \ll m$.

Previous authors (see, e.g., Refs. $[17,20]$ ) have typically related the nonrelativistic field $\psi$ to the relativistic field $\phi$ by using the relations

$$
\begin{aligned}
\phi(t, \mathbf{x}) & =\frac{1}{\sqrt{2 m}}\left[e^{-i m t} \psi(t, \mathbf{x})+e^{i m t} \psi^{*}(t, \mathbf{x})\right], \\
\pi(t, \mathbf{x}) & =-i \sqrt{\frac{m}{2}}\left[e^{-i m t} \psi(t, \mathbf{x})-e^{i m t} \psi^{*}(t, \mathbf{x})\right] .
\end{aligned}
$$

Note that the quantities in brackets in Eqs. (6a) and (6b) could have been written as $\operatorname{Re}\left[e^{-i m t} \psi\right]$ and $\operatorname{Im}\left[e^{-i m t} \psi\right]$, 
respectively, so the equations are independent. Equation (6b) is not ordinarily written explicitly, but this equation or something similar has to be assumed if $\psi(t, \mathbf{x})$ is to be uniquely defined. At any fixed time $t$, Eqs. (6a) and (6b), taken together, give a one-to-one mapping between the complex-valued $\psi(t, \mathbf{x})$ and the two real-valued functions $\phi(t, \mathbf{x})$ and $\pi(t, \mathbf{x})$. If we use Eqs. (3) and (6) to derive equations of motion for $\psi$, and then set $\lambda=0$ and neglect all rapidly oscillating terms (proportional to $e^{ \pm i m t}$ ), we find equations of motion consistent with the Lagrangian of Eq. (4). However, for the purpose of systematically obtaining the relativistic corrections to the nonrelativistic theory, we find it more convenient to start from a nonlocal field redefinition. In place of Eqs. (6), we write

$$
\begin{aligned}
& \phi(t, \mathbf{x})=\frac{1}{\sqrt{2 m}} \mathcal{P}^{-1 / 2}\left[e^{-i m t} \psi(t, \mathbf{x})+e^{i m t} \psi^{*}(t, \mathbf{x})\right], \\
& \pi(t, \mathbf{x})=-i \sqrt{\frac{m}{2}} \mathcal{P}^{1 / 2}\left[e^{-i m t} \psi(t, \mathbf{x})-e^{i m t} \psi^{*}(t, \mathbf{x})\right],
\end{aligned}
$$

where we have defined

$$
\mathcal{P} \equiv \sqrt{1-\frac{\nabla^{2}}{m^{2}}}
$$

Note that $m \mathcal{P}$ corresponds to the total energy of a free, relativistic particle. Equations (7) can be inverted to obtain an equation for $\psi$ in terms of $\phi$ and $\pi$ :

$\psi(t, \mathbf{x})=\sqrt{\frac{m}{2}} e^{i m t} \mathcal{P}^{1 / 2}\left(\phi(t, \mathbf{x})+\frac{i}{m} \mathcal{P}^{-1} \pi(t, \mathbf{x})\right)$.

Although our field redefinition in Eqs. (7) involves nonlocal operators, the new fields $\psi$ and $\psi^{*}$ are well behaved in the nonrelativistic limit, in which the operator $\mathcal{P}$ can be expanded in powers of $\nabla^{2} / \mathrm{m}^{2}$. The leading term matches the local definitions of Eq. (6). Furthermore, even though the $\psi$ field has a nonlocal relation to $\phi$, the $\psi$ field is local with respect to itself. When quantized, the commutator of $\psi(t, \mathbf{x})$ and $\psi^{*}(t, \mathbf{y})$ becomes

$$
\begin{aligned}
{\left[\psi(t, \mathbf{x}), \psi^{*}(t, \mathbf{y})\right] } \\
=\frac{m}{2} \mathcal{P}_{x}^{1 / 2} \mathcal{P}_{y}^{1 / 2}\left[\phi(t, \mathbf{x})-\frac{i}{m} \mathcal{P}_{x}^{-1} \pi(t, \mathbf{x}), \phi(t, \mathbf{y})\right. \\
\left.\quad+\frac{i}{m} \mathcal{P}_{y}^{-1} \pi(t, \mathbf{y})\right] \\
=\delta^{3}(\mathbf{x}-\mathbf{y})
\end{aligned}
$$

where the subscripts on $\mathcal{P}$ indicate the coordinates on which they act, and we assumed of course that $[\phi(t, \mathbf{x}), \pi(t, \mathbf{y})]=i \delta^{3}(\mathbf{x}-\mathbf{y})$. The simplicity of this result is a consequence of the fact that the nonlocal operators on the two lines of Eq. (7) are the inverses of each other.

Given Eqs. (3) and (9), it is straightforward to work out the equation of motion for $\psi(t, \mathbf{x})$ :

$$
\begin{aligned}
i \dot{\psi}= & m(\mathcal{P}-1) \psi \\
& +\frac{\lambda e^{i m t}}{4 ! m^{2}} \mathcal{P}^{-1 / 2}\left[e^{-i m t} \mathcal{P}^{-1 / 2} \psi+e^{i m t} \mathcal{P}^{-1 / 2} \psi^{*}\right]^{3} .
\end{aligned}
$$

A key step in this calculation, which motivates the nonlocal operator $\mathcal{P}$, is the calculation of the time derivative of $\phi+\frac{i}{m} \mathcal{P}^{-1} \pi$ :

$$
\begin{aligned}
\dot{\phi}+\frac{i}{m} \mathcal{P}^{-1} \dot{\pi} & =\pi+\frac{i}{m} \mathcal{P}^{-1}\left[\left(\nabla^{2}-m^{2}\right) \phi-\frac{1}{3 !} \lambda \phi^{3}\right] \\
& =\pi-i m \mathcal{P} \phi-\frac{i \lambda}{3 ! m} \mathcal{P}^{-1} \phi^{3} \\
& =-i m \mathcal{P}\left[\phi+\frac{i}{m} \mathcal{P}^{-1} \pi\right]-\frac{i \lambda}{3 ! m} \mathcal{P}^{-1} \phi^{3} .
\end{aligned}
$$

The definition of $\mathcal{P}$ was chosen so that the first term on the right-hand side of the last line above is proportional to $\phi+\frac{i}{m} \mathcal{P}^{-1} \pi \propto \psi$. If we used the local definition instead, the right-hand side would have also contained a term proportional to $e^{2 i m t} \psi^{*}$, which would lead to rapidly oscillating terms even in the free field theory $(\lambda=0)$. With the nonlocal field definition of Eqs. (7), the free theory leads to no rapidly oscillating terms.

It is for some purposes useful to write a Lagrangian density for the nonrelativistic formulation, so we note that the equation of motion in Eq. (11) can be derived from the Lagrangian density

$$
\begin{aligned}
\mathcal{L}= & \frac{i}{2}\left(\dot{\psi} \psi^{*}-\psi \dot{\psi^{*}}\right)-m \psi^{*}(\mathcal{P}-1) \psi \\
& -\frac{\lambda}{4 \cdot 4 ! m^{2}}\left[e^{-i m t} \mathcal{P}^{-1 / 2} \psi+e^{i m t} \mathcal{P}^{-1 / 2} \psi^{*}\right]^{4} .
\end{aligned}
$$

Note that the free field theory terms in Eq. (13), corresponding to $\lambda=0$, show a manifest global $U(1)$ symmetry, $\psi \rightarrow e^{i \theta} \psi$. This symmetry is associated with the conservation of particle number, which is exact in the free theory even when energies are relativistic.

To construct the corresponding Hamiltonian density, we explicitly decompose the field into its real and imaginary parts, $\psi \equiv \psi_{R}+i \psi_{I}$. The kinetic terms of the Lagrangian density then become

$$
\mathcal{L}_{\text {kinetic }}=\frac{i}{2}\left(\dot{\psi} \psi \psi^{*}-\psi \dot{\psi}^{*}\right)=\dot{\psi}_{R} \psi_{I}-\psi_{R} \dot{\psi}_{I} .
$$

If we take $\psi_{R}$ to be the canonical field, then $\psi_{I}$ will become the canonical momentum $\partial \mathcal{L} / \partial \dot{\psi}_{R}$. If we proceeded directly to construct the Hamiltonian density in the standard 
way, $\mathcal{H}=\pi \dot{\psi}_{R}-\mathcal{L}$, the second term on the right of Eq. (14) would be rewritten as $-\psi_{R} \dot{\psi}_{I}=-\psi_{R} \dot{\pi}$. This, however, would take us outside the standard Hamiltonian procedure, in which the Hamiltonian is assumed to be a function of the fields and their canonical momenta, but not the time derivatives of canonical momenta. To avoid this problem, we can add a total time derivative to the Lagrangian density, which does not change the equations of motion. So we replace $\mathcal{L}_{\text {kinetic }}$ by

$$
\mathcal{L}_{\text {kinetic }}^{\prime}=\mathcal{L}_{\text {kinetic }}+\frac{d}{d t}\left(\psi_{R} \psi_{I}\right)=2 \dot{\psi}_{R} \psi_{I}
$$

To absorb the factor of 2 we find it convenient to define the canonical field $\psi_{c}$ to be

$$
\psi_{c}(t, \mathbf{x}) \equiv \sqrt{2} \psi_{R}(t, \mathbf{x}),
$$

from which it follows that

$$
\pi_{c}(t, \mathbf{x}) \equiv \frac{\partial \mathcal{L}}{\partial \dot{\psi}_{c}(t, \mathbf{x})}=\sqrt{2} \psi_{I}(t, \mathbf{x}) .
$$

Thus, we have

$$
\psi(t, \mathbf{x})=\frac{1}{\sqrt{2}}\left(\psi_{c}(t, \mathbf{x})+i \pi_{c}(t, \mathbf{x})\right) .
$$

The Hamiltonian density is then given by

$$
\begin{aligned}
\mathcal{H} & =\pi_{c} \dot{\psi}_{c}-\mathcal{L} \\
& =m \psi^{*}(\mathcal{P}-1) \psi+\frac{\lambda}{4 \cdot 4 ! m^{2}}\left[e^{-i m t} \mathcal{P}^{-1 / 2} \psi+e^{i m t} \mathcal{P}^{-1 / 2} \psi^{*}\right]^{4},
\end{aligned}
$$

where $\psi$ is given by Eq. (18). The canonical quantization of this Hamiltonian would give

$$
\left[\psi_{c}(t, \mathbf{x}), \pi_{c}(t, \mathbf{y})\right]=i \delta^{3}(\mathbf{x}-\mathbf{y}),
$$

which is equivalent to Eq. (10). The discussion here has shown that the Hamiltonian of Eq. (19) gives the correct equation of motion for the field $\psi(t, \mathbf{x})$. In Appendix A, we show that the transformation from $\phi(t, \mathbf{x})$ to $\psi(t, \mathbf{x})$ can be constructed as a canonical transformation, which guarantees that the canonical commutators will be preserved, as we have found.

Our findings are in contrast with the recent claim in Ref. [21], in which the authors found it problematic to derive a Lagrangian for a complex, nonrelativistic field from a real-valued relativistic one. Based on the canonical transformation we have explicitly formulated, we do not believe there should be any difficulty in constructing $\mathcal{L}(\psi, \dot{\psi})$. It is interesting to note that we may obtain the Lagrangian in Eq. (13) directly from the Lagrangian in
Eq. (1) by the following procedure. Replace $\dot{\phi}$ in the Lagrangian by an auxiliary field using $\chi=\dot{\phi}$, and impose the relation via a Lagrange multiplier: $\mathcal{L}[\phi, \dot{\phi}] \rightarrow$ $\mathcal{L}[\phi, \chi]+\zeta(\chi-\dot{\phi})$, where $\zeta$ is a Lagrange multiplier. We next note that $\chi$ is a nondynamical variable in the resulting Lagrangian. We remove it from the theory by varying $\mathcal{L}$ with respect to $\chi$, which yields an expression for $\chi$, which we then substitute back into $\mathcal{L}$. We use similar relations as in Eqs. (7) to relate the set $\{\phi, \zeta\}$ (instead of $\{\phi, \pi\}$ ) to the set $\left\{\psi, \psi^{*}\right\}$, and substitute the relations back into the Lagrangian.

We close this section with a final observation about the field redefinitions in Eqs. (7), compared to the traditional relation in Eqs. (6). The usual relation of Eqs. (6) has the advantage of being local, but the resulting Lagrangianeven for free fields - would no longer preserve the manifest $U(1)$ symmetry, thereby obscuring the fact that such models should conserve particle number. Moreover, if we had used Eqs. (6), the fast oscillatory factors $\left(e^{ \pm i m t}\right)$, which are absent in the free field theory using our nonlocal formulation, would have appeared in the free-field Lagrangian. We build upon these advantages of the relations in Eqs. (7) in the following section.

\section{EFFECTIVE FIELD THEORY IN THE NONRELATIVISTIC LIMIT}

Given the Lagrangian and equation of motion for $\psi$ in Eqs. (13) and (11), we may now consider the effective description for such a model in the nonrelativistic limit. We aim to take the nonrelativistic limit in a way that enables us to incorporate relativistic corrections systematically. One step will obviously be to expand the nonlocal operator $\mathcal{P}$ in powers of $\nabla^{2} / \mathrm{m}^{2}$. However, we must take additional steps in order to recover an appropriate description in the nonrelativistic limit. In particular, we must find some way to incorporate the effects of fast-oscillating terms on the behavior of the slowly varying field.

Consider, as a first step, the equation of motion of Eq. (11) in the limit in which we may ignore all higher spatial-derivative terms arising from the expansion of $\mathcal{P}$. Then we find

$$
\begin{aligned}
i \dot{\psi} \simeq & -\frac{1}{2 m} \nabla^{2} \psi+\frac{\lambda}{8 m^{2}}|\psi|^{2} \psi \\
& +\frac{\lambda}{4 ! m^{2}}\left[e^{-2 i m t} \psi^{3}+e^{4 i m t} \psi^{* 3}+3 e^{2 i m t}|\psi|^{2} \psi^{*}\right] .
\end{aligned}
$$

The first two terms on the right-hand side consist of the usual terms in the Schrödinger equation for a model incorporating self-interactions $[17,20]$. The remaining terms are usually neglected in the nonrelativistic limit, because they are proportional to fast-oscillating factors, and in the limit of large $m$, such terms might be expected to average to zero if the system were observed over timescales 
$\Delta t \gg m^{-1}$. However, such oscillatory terms must be treated with care. Suppose, for example, that the full solution of Eq. (21) consists of a slowly varying contribution $\left(\psi_{s}\right)$ and a small term that does not vary slowly: $\psi=\psi_{s}+\delta \psi e^{2 i m t}$. Inserting this ansatz back into Eq. (21), keeping only terms up to linear order in $\delta \psi$, and retaining terms that vary in time more slowly than $e^{ \pm i m t}$, we obtain

$$
\begin{aligned}
i \dot{\psi}_{s} \simeq & -\frac{1}{2 m} \nabla^{2} \psi_{s}+\frac{\lambda}{8 m^{2}}\left|\psi_{s}\right|^{2} \psi_{s} \\
& +\frac{\lambda}{8 m^{2}} \psi_{s}\left(\psi_{s} \delta \psi+2 \psi_{s}^{*} \delta \psi^{*}\right)+\ldots
\end{aligned}
$$

We find that the fast-oscillating portion of the solution $(\delta \psi)$ backreacts on the slowly varying portion $\left(\psi_{s}\right)$, contributing to the dynamics of $\psi_{s}$ in a nontrivial way. (Cf. Refs. [18,19].) This simple example illustrates that we must take into account the fast-oscillating contributions of the full solution to obtain an effective description of the slowly varying portion. The removal of the fast-oscillating contributions is analogous to integrating out the highfrequency components of a field in a path integral.

We construct a perturbative approach with which to account for the contribution of the fast-oscillating terms to the time evolution of the slowly varying portion of the field $\psi$. In general there are three small quantities to consider for our perturbative treatment. For any quantity $F(t, \mathbf{x})$, we may consider spatial variations compared to the length-scale $\mathrm{m}^{-1}$; time variation compared to the timescale $\mathrm{m}^{-1}$; and self-interactions mediated by the coupling constant $\lambda$. We parametrize the magnitude of spatial and temporal variations as

$$
\frac{\nabla^{2} F}{m^{2}} \sim \epsilon_{x} F, \quad \frac{\dot{F}}{m} \sim \epsilon_{t} F .
$$

For weakly interacting systems in the nonrelativistic limit, we assume that $\epsilon_{x}, \epsilon_{t}, \lambda \ll 1$. Given our nonlocal field redefinition in terms of the operator $\mathcal{P}$, we may manipulate quantities to arbitrary order in $\epsilon_{x}$ during intermediate steps and expand the $\mathcal{P}$ operators to the desired order at the end. We may then Taylor expand various quantities in powers of $\epsilon_{t}$ and $\lambda$ and iteratively track their effects on the slowly varying portion of the field. Upon expanding the $\mathcal{P}$ operators, we may use the equation of motion (at appropriate, iterative order) to relate higher-order terms in $\epsilon_{t}$ to corresponding higher-order terms in $\epsilon_{x}$ and $\lambda$. In the end this yields a controlled expansion in two small parameters, $\epsilon_{x}$ and $\lambda$.

We construct the solution for $\psi(t, \mathbf{x})$ in the form of an expansion in an infinite series of harmonics,

$$
\psi(t, \mathbf{x})=\sum_{\nu=-\infty}^{\infty} \psi_{\nu}(t, \mathbf{x}) e^{i \nu m t},
$$

where each $\psi_{\nu}(t, \mathbf{x})$ will be slowly varying on a timescale of order $m^{-1}$. We label the mode with $\nu=0$ as the slowly varying contribution to the field,

$$
\psi_{\nu=0}(t, \mathbf{x}) \equiv \psi_{s}(t, \mathbf{x})
$$

We assume that in the nonrelativistic limit, $\left|\psi-\psi_{s}\right| \ll\left|\psi_{s}\right|$.

We may rewrite the equation of motion for $\psi$ in Eq. (11) in the form

$$
i \dot{\psi}(t, \mathbf{x})=m(\mathcal{P}-1) \psi(t, \mathbf{x})+\frac{\lambda}{4 ! m^{2}} \tilde{G}(t, \mathbf{x}),
$$

where we have defined

$$
\tilde{G}(t, \mathbf{x}) \equiv \mathcal{P}^{-1 / 2} e^{i m t}\left[e^{-i m t} \mathcal{P}^{-1 / 2} \psi+e^{i m t} \mathcal{P}^{-1 / 2} \psi^{*}\right]^{3} .
$$

We expand $\tilde{G}$ in a series akin to the expansion for $\psi$ :

$$
\tilde{G}(t, \mathbf{x})=\sum_{\nu=-\infty}^{\infty} \tilde{G}_{\nu}(t, \mathbf{x}) e^{i \nu m t} .
$$

For a given mode $\nu$, Eq. (26) then takes the form

$$
i \dot{\psi}_{\nu}-\nu m \psi_{\nu}=m(\mathcal{P}-1) \psi_{\nu}+\frac{\lambda}{4 ! m^{2}} \tilde{G}_{\nu}
$$

with

$$
\begin{aligned}
\tilde{G}_{\nu}(t, \mathbf{x})= & \mathcal{P}^{-1 / 2} \sum_{\mu, \mu^{\prime}}\left\{\Psi_{\mu} \Psi_{\mu^{\prime}} \Psi_{2+\nu-\mu-\mu^{\prime}}+\Psi_{\mu}^{*} \Psi_{\mu^{\prime}}^{*} \Psi_{4-\nu-\mu-\mu^{\prime}}^{*}\right. \\
& \left.+3 \Psi_{\mu} \Psi_{\mu^{\prime}} \Psi_{\mu+\mu^{\prime}-\nu}^{*}+3 \Psi_{\mu}^{*} \Psi_{\mu^{\prime}}^{*} \Psi_{\nu-2+\mu+\mu^{\prime}}\right\},
\end{aligned}
$$

where we have made use of the convenient notation

$$
\Psi_{\nu}(t, \mathbf{x}) \equiv \mathcal{P}^{-1 / 2} \psi_{\nu}(t, \mathbf{x})
$$

For $\nu=0$, Eq. (29) gives us the equation obeyed by $\psi_{s}(t, \mathbf{x})$, which defines the low-energy effective field theory that we seek. However, to evaluate $\tilde{G}_{\nu}$, we need to (perturbatively) calculate $\psi_{\nu}(t, \mathbf{x})$ for all other values of $\nu$. Before proceeding, we note that for each $\nu$, the mode $\psi_{\nu}$ carries a definite charge. In particular, if we assign charges $Q_{\nu}=1-\nu$ to $\psi_{\nu}$ and $\bar{Q}_{\nu}=-Q_{\nu}$ to $\psi_{\nu}^{*}$, then total charge remains conserved because all terms in Eq. (29) carry the same charge. This implies that the mode of interest, $\psi_{s}$, carries a conserved charge, and hence we expect the effective field theory governing $\psi_{s}$ to obey a global $U(1)$ symmetry, to all orders in the perturbative expansion, in spite of the fact that the exact theory violates this symmetry. Below we will explicitly confirm that particle number conservation is exact for the leading perturbative corrections. 
Equation (29) is a first-order differential equation in time, which in principle defines $\psi_{\nu}(t, \mathbf{x})$ up to an arbitrary function of $\mathbf{x}$. However, as long as we can construct a function $\psi_{\nu}(t, \mathbf{x})$ which satisfies Eq. (29), then the full series of Eq. (24) will satisfy the equation of motion, Eq. (11), so it will be sufficient for us to construct a particular solution to Eq. (29). To do so, we multiply both sides of Eq. (29) by $\mathcal{P}^{-1 / 2}$ and rearrange terms to write

$$
\Psi_{\nu}=-\frac{i}{m} \Gamma_{\nu} \dot{\Psi}_{\nu}+\lambda G_{\nu}
$$

where we have defined

$$
\Gamma_{\nu} \equiv(1-\nu-\mathcal{P})^{-1}
$$

and

$$
\begin{aligned}
G_{\nu}(t, \mathbf{x})= & \frac{\Gamma_{\nu} \mathcal{P}^{-1 / 2}}{4 ! m^{3}} \tilde{G}_{\nu}(t, \mathbf{x}) \\
= & \frac{\Gamma_{\nu} \mathcal{P}^{-1}}{4 ! m^{3}} \sum_{\mu, \mu^{\prime}}\left\{\Psi_{\mu} \Psi_{\mu^{\prime}} \Psi_{2+\nu-\mu-\mu^{\prime}}+\Psi_{\mu}^{*} \Psi_{\mu^{\prime}}^{*} \Psi_{4-\nu-\mu-\mu^{\prime}}^{*}\right. \\
& \left.+3 \Psi_{\mu} \Psi_{\mu^{\prime}} \Psi_{\mu+\mu^{\prime}-\nu}^{*}+3 \Psi_{\mu}^{*} \Psi_{\mu^{\prime}}^{*} \Psi_{\nu-2+\mu+\mu}\right\} .
\end{aligned}
$$

Equation (32) holds to any order in $\epsilon_{x}, \epsilon_{t}$, and $\lambda$. We note, however, that the first term on the right-hand side is suppressed relative to $\Psi_{\nu}$ by a factor of $\epsilon_{t}$ and the second term is suppressed relative to $G_{\nu}$ by a factor of $\lambda$, so we may treat the right-hand side as a perturbative source for $\Psi_{\nu}$. Therefore we can solve Eq. (32) iteratively, starting with the zeroth-order approximation

$$
\Psi_{\nu}^{(0)}(t, \mathbf{x})= \begin{cases}\Psi_{s}(t, \mathbf{x}) & \text { if } \nu=0 \\ 0 & \text { if } \nu \neq 0\end{cases}
$$

We denote by $\Psi_{\nu}^{(n)}$ the correction to $\Psi_{\nu}$ obtained at the $n$th iteration, which in our construction will always be proportional to a total of $n$ powers of the small quantities $\epsilon_{t}$ and $\lambda$. We expand the modes with $\nu \neq 0$ in the series

$$
\Psi_{\nu}(t, \mathbf{x})=\sum_{n=0}^{\infty} \Psi_{\nu}^{(n)}(t, \mathbf{x}) \quad \text { for } \nu \neq 0
$$

where the terms $\Psi_{\nu}^{(n)}$ will be determined iteratively, as described below, and $\Psi_{0}^{(n)} \equiv 0$ for $n>0$, since we are not expanding $\Psi_{0} \equiv \Psi_{s}$ in a power series. We also expand

$$
G_{\nu}(t, \mathbf{x})=\sum_{n=0}^{\infty} G_{\nu}^{(n)}(t, \mathbf{x})
$$

where $G_{\nu}^{(n)}(t, \mathbf{x})$ consists of all terms in Eq. (34) which are proportional to the $n$th power of the small quantities $\epsilon_{t}$ and $\lambda$. From Eq. (32), we have at first order

$$
\Psi_{\nu}^{(1)}=\lambda G_{\nu}^{(0)} \quad \text { for } \nu \neq 0
$$

and for higher orders we have

$\Psi_{\nu}^{(n)}=-\frac{i}{m} \Gamma_{\nu} \dot{\Psi}_{\nu}^{(n-1)}+\lambda G_{\nu}^{(n-1)}$ for $\nu \neq 0$ and $n>1$.

The first term on the right-hand side is suppressed relative to $\Psi_{\nu}^{(n-1)}$ by $\epsilon_{t}$, and the second term is suppressed relative to $G_{\nu}^{(n-1)}$ by $\lambda$. Thus, from Eq. (39), we see by induction that $\Psi_{\nu}^{(n)}$ will have a total power in the small quantities $\epsilon_{t}$ and $\lambda$ of exactly $n$.

Our aim is to calculate the effective equation of motion for $\psi_{s}$, which is given by Eqs. (29) and (34) as

$$
i \dot{\psi}_{s}=m(\mathcal{P}-1) \psi_{s}+m \lambda \Gamma_{0}^{-1} \mathcal{P}^{1 / 2} G_{0},
$$

with $G_{0}$ expanded to some perturbative order, as in Eq. (37). Working to order $n=1$ we find

$$
\begin{aligned}
G_{\nu}^{(0)}= & \frac{1}{\lambda} \Psi_{\nu}^{(1)}=\frac{\Gamma_{\nu} \mathcal{P}^{-1}}{4 ! m^{3}}\left\{\Psi_{s}^{3} \delta_{\nu,-2}+\Psi_{s}^{* 3} \delta_{\nu, 4}+3\left|\Psi_{s}\right|^{2} \Psi_{s}^{*} \delta_{\nu, 2}\right. \\
& \left.+3\left|\Psi_{s}\right|^{2} \Psi_{s} \delta_{\nu, 0}\right\}, \\
G_{\nu}^{(1)}= & \frac{3 \Gamma_{\nu} \mathcal{P}^{-1}}{4 ! m^{3}}\left\{\Psi_{s}^{2} \Psi_{2+\nu}^{(1)}+\Psi_{s}^{* 2} \Psi_{4-\nu}^{(1) *}+\Psi_{s}^{2} \Psi_{-\nu}^{(1) *}\right. \\
& \left.+2\left|\Psi_{s}\right|^{2} \Psi_{\nu}^{(1)}+\Psi_{s}^{* 2} \Psi_{\nu-2}^{(1)}+2\left|\Psi_{s}\right|^{2} \Psi_{2-\nu}^{(1) *}\right\},
\end{aligned}
$$

where $\delta_{i, j}$ is the Kronecker delta function and, as discussed after Eq. (36), we set $\Psi_{0}^{(n)}=0$. Using the expressions in Eq. (41), we may expand Eq. (40) to order $n=1$, which yields

$$
\begin{aligned}
i \dot{\psi}_{s}= & m(\mathcal{P}-1) \psi_{s}+\frac{\lambda \mathcal{P}^{-1 / 2}}{8 m^{2}}\left|\Psi_{s}\right|^{2} \Psi_{s} \\
& +\frac{3 \lambda^{2} \mathcal{P}^{-1 / 2}}{(4 !)^{2} m^{5}}\left\{3 \Psi_{s}^{2} \Gamma_{2} \mathcal{P}^{-1}\left(\left|\Psi_{s}\right|^{2} \Psi_{s}^{*}\right)+\Psi_{s}^{* 2} \Gamma_{4} \mathcal{P}^{-1}\left(\Psi_{s}^{3}\right)\right. \\
& \left.+\Psi_{s}^{* 2} \Gamma_{-2} \mathcal{P}^{-1}\left(\Psi_{s}^{3}\right)+6\left|\Psi_{s}\right|^{2} \Gamma_{2} \mathcal{P}^{-1}\left(\left|\Psi_{s}\right|^{2} \Psi_{s}\right)\right\} \\
& +\mathcal{O}\left[\lambda^{3}, \epsilon_{t}^{3}, \lambda^{2} \epsilon_{t}, \lambda \epsilon_{t}^{2}\right]
\end{aligned}
$$

We may now expand the $\mathcal{P}$ operators to order $\epsilon_{x}^{2} \sim \nabla^{4} / m^{4}$, which yields 


$$
\begin{aligned}
i \dot{\psi}_{s} \simeq & -\frac{1}{2 m} \nabla^{2} \psi_{s}+\frac{\lambda}{8 m^{2}}\left|\psi_{s}\right|^{2} \psi_{s} \\
& -\frac{1}{8 m^{3}} \nabla^{4} \psi_{s}+\frac{\lambda}{32 m^{4}}\left[\psi_{s}^{2} \nabla^{2} \psi_{s}^{*}+2\left|\psi_{s}\right|^{2} \nabla^{2} \psi_{s}\right. \\
& \left.+\nabla^{2}\left(\left|\psi_{s}\right|^{2} \psi_{s}\right)\right]-\frac{17 \lambda^{2}}{768 m^{5}}\left|\psi_{s}\right|^{4} \psi_{s} \\
& +\mathcal{O}\left[\lambda^{3}, \epsilon_{t}^{3}, \epsilon_{x}^{3}, \lambda^{2} \epsilon_{t}, \lambda^{2} \epsilon_{x}, \lambda \epsilon_{t}^{2}, \lambda \epsilon_{x}^{2}, \lambda \epsilon_{t} \epsilon_{x}, \epsilon_{t} \epsilon_{x}^{2}, \epsilon_{t}^{2} \epsilon_{x}\right] .
\end{aligned}
$$

To obtain Eq. (43), we only needed to calculate the $\Psi_{\nu}^{(n)}$ up to $n=1$, so it was sufficient to use Eq. (38), and the time derivative in Eq. (39) never appeared. But of course if we continued to the next order, the time derivative of $\Psi_{\nu}^{(n-1)}$ would appear. The iteration of this procedure will lead to time derivatives higher than the first, which will give at least the appearance of introducing new degrees of freedom (d.o.f.). There exists, however, a well-defined procedure for treating these higher time derivatives perturbatively, so that no new d.o.f. are introduced. We illustrate these techniques in Appendices B and C. The higher time derivatives can be removed later, but in the context of the iteration procedure described here, they can be eliminated at each iteration. To do this, start by writing $\Psi_{\nu}^{(n-1)}$ in terms of $\psi_{s}$. For $n=2$, this would be the top line of Eq. (41). At each stage, we will be able to express $\Psi_{\nu}^{(n-1)}$ in a perturbative expansion, with all terms up to a total power of $n-1$ in the small quantities $\epsilon_{t}$ and $\lambda$. Then we can differentiate this expression with respect to $t$, evaluating $\dot{\psi}_{s}$ according to Eq. (40). Even though $\Psi_{\nu}^{(n-1)}$ has a total power of $n-1$ in the small quantities $\epsilon_{t}$ and $\lambda$, its time derivative can be calculated up to one power higher, since the time derivative brings an extra factor of $\epsilon_{t}$. Thus the first term on the right-hand side of Eq. (39) can be evaluated as a term with no time derivatives, and with a total power of $n$ in the small quantities $\epsilon_{t}$ and $\lambda$, which is exactly what is wanted.

The first line of Eq. (43) is the usual Schrödinger equation for a self-interacting scalar field in the nonrelativistic limit, and the other lines include the lowestorder relativistic corrections. The terms that include the Laplacian operator come from expanding the operator $\mathcal{P}$, and the final term comes from the contribution of the fastoscillating terms $\psi_{\nu}^{(1)}$ (for $\nu \neq 0$ ). Given the equation of motion (43), we can find an effective Lagrangian which produces this equation of motion:

$$
\begin{aligned}
\mathcal{L}_{\text {eff }}= & \frac{i}{2}\left(\dot{\psi}_{s} \psi_{s}^{*}-\psi_{s} \dot{\psi}_{s}^{*}\right)-\frac{1}{2 m} \nabla \psi_{s} \nabla \psi_{s}^{*}-\frac{\lambda}{16 m^{2}}\left|\psi_{s}\right|^{4} \\
& +\frac{1}{8 m^{3}} \nabla^{2} \psi_{s} \nabla^{2} \psi_{s}^{*}-\frac{\lambda}{32 m^{4}}\left|\psi_{s}\right|^{2}\left(\psi_{s}^{*} \nabla^{2} \psi_{s}+\psi_{s} \nabla^{2} \psi_{s}^{*}\right) \\
& +\frac{17 \lambda^{2}}{9 \times 2^{8} m^{5}}\left|\psi_{s}\right|^{6} .
\end{aligned}
$$

We note that a single self-interaction term in the relativistic Lagrangian (in this case, $\lambda \phi^{4} / 4$ !) yields more than one interaction term in the nonrelativistic effective theory. In particular, the effective Lagrangian for $\psi_{s}$ in Eq. (44) includes interaction terms that yield both $2 \rightarrow 2$ scattering and $3 \rightarrow 3$ scattering; including more terms in the iterative expansion for $\psi_{\nu}$ (with $\nu \neq 0$ ) would yield operators in $\mathcal{L}_{\text {eff }}$ for each $n \rightarrow n$ scattering, for $n>1$. On the other hand, $\mathcal{L}_{\text {eff }}$ obeys a global $U(1)$ symmetry, a feature which holds to all orders in the perturbative expansion, as discussed after Eq. (31). Thus, particle number is conserved to all orders in the nonrelativistic effective field theory. A process that may occur in the relativistic theory, such as $4 \rightarrow 2$ scattering in which four low-energy particles annihilate to produce two relativistic ones, involves energies $E>m$ and hence lies beyond the range of validity of $\mathcal{L}_{\text {eff }}$ in Eq. (44).

We have obtained the effective theory in Eq. (44) starting from the nonlocal field redefinition of Eqs. (7). Our procedure was relatively straightforward because the equation of motion in the free-field limit does not contain fastoscillating factors, and all of the spatial-derivative operators may be manipulated in terms of the nonlocal operator $\mathcal{P}$. Whereas these steps simplify the calculation when working with the nonlocal field redefinition of Eqs. (7), one may follow similar steps to derive an equivalent effective field theory starting from the local field redefinition of Eqs. (6), as we demonstrate in Appendix B.

We may apply Eqs. (43) and (44) to the case of QCD axions in the nonrelativistic limit, and compare our effective description with other recent treatments $[17,20]$. We begin with the usual relativistic potential,

$$
V(\phi)=\Lambda^{4}\left[1-\cos \left(\phi / f_{a}\right)\right]
$$

where $\Lambda \sim 0.1 \mathrm{GeV}$ is associated with the QCD scale, and $f_{a}$ sets the scale for Peccei-Quinn symmetry breaking; we expect $f_{a} \sim 10^{11}-10^{12} \mathrm{GeV}$ for the typical Peccei-Quinn model [14-17]. For field values well below $f_{a}$, we may expand $V(\phi)$ as

$$
V(\phi)=\frac{1}{2} m^{2} \phi^{2}+\frac{\lambda}{4 !} \phi^{4}+\mathcal{O}\left[\left(\phi / f_{a}\right)^{6}\right],
$$

where $m=\Lambda^{2} / f_{a}$ and $\lambda=-\Lambda^{4} / f_{a}^{4}<0$. Given expected values for $\Lambda$ and $f_{a}$, these correspond to $m \sim 10^{-4}-10^{-5} \mathrm{eV}$ and $|\lambda| \sim 10^{-48}-10^{-52}$.

Remarkably, the final term in Eq. (43), proportional to $\lambda^{2}$, exactly matches the corresponding term found in Ref. [20], following quite a different procedure. (In Ref. [20], the authors fixed the coefficients in a series expansion for the low-energy effective potential by calculating scattering amplitudes for various $n$-body scattering processes in the full, relativistic theory, and then took the low-energy limit of those amplitudes.) On the other hand, 
the analysis in Ref. [20] does not capture the relativistic corrections present in Eq. (43), proportional to $\nabla^{4}$ and $\lambda \nabla^{2}$. We may compare magnitudes for the $\lambda \nabla^{2}$ and $\lambda^{2}$ terms:

$$
\begin{aligned}
& \left(\frac{17 \lambda^{2}\left|\psi_{s}\right|^{4} \psi_{s}}{768 m^{5}}\right)\left(\frac{32 m^{2}}{\lambda\left|\psi_{s}\right|^{2} \nabla^{2} \psi_{s}}\right) \\
& \quad=\mathcal{O}(1)\left(\frac{\lambda\left|\psi_{s}\right|^{2} \psi_{s}}{8 m^{2}}\right)\left(\frac{2 m}{\nabla^{2} \psi_{s}}\right) \sim \frac{\lambda \mathcal{N}}{m^{3} v^{2}},
\end{aligned}
$$

where $\mathcal{N}=\left|\psi_{s}\right|^{2}$ is the axion number density, and we have used $\nabla^{2} / m^{2} \sim v^{2}$. Thus we see that whenever the contributions arising from kinetic and potential energy to the zeroth-order equation of motion are of comparable magnitude to each other - that is, the two terms on the right-hand side of the top line of Eq. (43) - then the leading-order correction terms, on the second and third lines of Eq. (43), will also be of comparable magnitude to each other. In such situations, it is inconsistent to retain (for example) the $\lambda^{2}$ corrections while neglecting the $\lambda \nabla^{2}$ corrections. Put another way, one must retain each of the corrections on the second and third lines of Eq. (43) for situations in which the axions have virialized, with comparable (time-averaged) values of kinetic and potential energy [22]. We further note that in Ref. [20], the term $m\left|\psi_{s}\right|^{2}$ appears in their low-energy Hamiltonian [Eqs. (26) and (27) of their paper], whereas we find no such term in ours. As we show in Appendix A [see Eqs. (A3) and (A11)], the $m\left|\psi_{s}\right|^{2}$ term is exactly canceled by a compensating term arising from the canonical transformation.

We may also compare our results in Eq. (43) with the recent calculation in Ref. [11]. Superficially our equations of motion appear to disagree at order $\lambda^{2}$. However, as demonstrated in Appendix C, our results are completely consistent (at least to this perturbative order), as can be shown by performing a nonlinear field redefinition.

Finally, we note that if one considers corrections from modes $\psi_{\nu}^{(n)}$ with $\nu \neq 0$ and $n \geq 2$, then one will generically find higher-order time-derivative terms in the effective equation of motion for $\psi_{s}$, as discussed after Eq. (43). This is because the mode functions for fast-oscillating terms $\psi_{\nu}^{(j)}$ depend, in general, on both $\psi_{\nu}^{(i)}$ and $\dot{\psi}_{\nu}^{(i)}$ for $i<j$. Such higher-order time derivatives do not introduce unphysical d.o.f.-after all, the full, relativistic theory remains well-behaved. Rather, such higher-order time derivatives typically arise in perturbative expansions for low-energy effective field theories, and may be removed systematically by applying the equations of motion at an appropriate perturbative order [25]. This procedure corresponds to excluding certain solutions of the low-energy effective theory that could not have been considered perturbations around the zeroth-order equations of motion, and hence remains consistent with the spirit of constructing effective field theories [25-27].

\section{CONCLUSIONS}

In this paper we have developed a self-consistent framework for obtaining an effective field theory to describe the nonrelativistic limit of a relativistic field theory. The lowestorder corrections to the ultranonrelativistic limit arise both from expanding the kinetic energy as well as from incorporating the backreaction from fast-oscillating terms on the dominant, slowly varying portion of the field. Our results are largely consistent with the complementary analyses of Refs. [11,20]. Our approach is perhaps simpler, working directly with the equations of motion in the nonrelativistic limit. In addition, our approach incorporates nontrivial relativistic corrections in a systematic way, which had been neglected in previous analyses. These additional terms may be comparable in magnitude to the other, known terms in various physical situations of interest.

Rather than begin with the usual relation of Eqs. (6) between the real, relativistic scalar field and the complex, nonrelativistic field, we introduce a fairly simple, nonlocal field redefinition, as in Eqs. (7). This new field redefinition considerably simplifies the treatment for free fields, and enables us to construct an iterative, perturbative procedure in the presence of interactions.

Other than imposing the standard commutation relations, our treatment relies on manipulating classical fields. Even so, we believe our formalism captures the relevant dynamics of the low-energy effective quantum field theoryincluding higher-order corrections in the coupling constant $\lambda$. The tree-level diagrams of the quantum theory directly reflect the equations of motion of the classical theory, and we would expect that any low-energy effective Lagrangian that produces the correct tree-level diagrams will also produce the correct loop diagrams. In our formalism we find that particle number is conserved to all orders in the nonrelativistic, perturbative description; an interesting question is how best to incorporate particle-number-violating processes in a low-energy effective description, in a self-consistent way. (Cf. Refs. [11,28].)

We have focused on the dynamics of a single real, selfinteracting scalar field. Applications of interest, which we intend to explore in future work, include the behavior of axion dark matter in galactic halos (building on Refs. [17,29] and references therein), as well as in hypothetical axion stars [28,30-36], for which one must supplement our treatment here to incorporate gravitational interactions. It would also be interesting to extend the approach developed here to other types of fields, such as the Dirac field.

\section{ACKNOWLEDGMENTS}

It is a pleasure to thank Mustafa Amin, Mark Hertzberg, Chanda Prescod-Weinstein, Iain Stewart, and Masaki Yamada for helpful discussions, and to acknowledge support from the Center for Theoretical Physics at MIT. 
This work is supported in part by the U.S. Department of Energy under grant Contract No. DE-SC0012567.

Note added.-As we were finalizing revisions to this paper, Ref. [37] appeared, which reaches similar conclusions regarding relations among various approaches to the low-energy effective descriptions of a given relativistic quantum field theory.

\section{APPENDIX A: CANONICAL DERIVATION OF THE FIELD REDEFINITION}

Our calculations have been based on the field redefinition of Eqs. (7), and their inverse given by Eq. (9). In Eq. (13) we constructed a Lagrangian that gives the correct equation of motion for $\psi$, but in this Appendix we show how to construct the field redefinition as a canonical transformation. Canonical transformations are guaranteed to preserve the Poisson bracket relations, which become the commutators upon quantization.

We start by adopting the real part of $\psi$ as the new canonical field $\psi_{c}$, but we allow a normalization factor $\mathcal{C}_{1}$ that can later be adjusted for convenience:

$$
\psi_{c}=\mathcal{C}_{1} \psi_{R},
$$

where $\psi \equiv \psi_{R}+i \psi_{I}$. The canonical momentum conjugate to $\psi_{c}$ will be called $\pi_{c}$, which we expect to be proportional to $\psi_{I}$ :

$$
\pi_{c}=\mathcal{C}_{2} \psi_{I}
$$

$\phi$ and $\pi$ will be used to denote the original relativistic field and its canonical conjugate. To describe the canonical transformation, we adapt the discrete-variable formalism of Goldstein, Poole, and Safko [38] to the case of continuous fields. The canonical transformation is then defined in terms of a generating functional $F_{2}\left[\phi, \pi_{c}, t\right]$, and the canonical transformation is described by

$\pi(x)=\frac{\delta F_{2}}{\delta \phi(x)}, \quad \psi_{c}(x)=\frac{\delta F_{2}}{\delta \pi_{c}(x)}, \quad H_{\text {new }}=H+\frac{\partial F_{2}}{\partial t}$,

where the partial derivatives in Ref. [38] have been replaced by functional derivatives. To try to match these equations, we first rewrite the transformation in terms of the real and imaginary parts of $\psi$ :

$$
\begin{aligned}
& \phi(t, \mathbf{x})=\sqrt{\frac{2}{m}} \mathcal{P}^{-1 / 2}\left[\cos (m t) \psi_{R}(t, \mathbf{x})+\sin (m t) \psi_{I}(t, \mathbf{x})\right], \\
& \pi(t, \mathbf{x})=\sqrt{2 m} \mathcal{P}^{1 / 2}\left[\cos (m t) \psi_{I}(t, \mathbf{x})-\sin (m t) \psi_{R}(t, \mathbf{x})\right] .
\end{aligned}
$$

We can then algebraically manipulate these equations to find expressions for $\pi(x)$ and $\psi_{c}(x)$ in terms of $\phi$ and $\pi_{c}$, to compare with Eqs. (A3):

$$
\begin{aligned}
\psi_{c}(t, \mathbf{x})= & \mathcal{C}_{1}\left[\sqrt{\frac{m}{2}} \mathcal{P}^{1 / 2} \sec (m t) \phi(t, \mathbf{x})\right. \\
& \left.-\frac{1}{\mathcal{C}_{2}} \tan (m t) \pi_{c}(t, \mathbf{x})\right], \\
\pi(t, \mathbf{x})= & \frac{1}{\mathcal{C}_{2}} \sqrt{2 m} \mathcal{P}^{1 / 2} \sec (m t) \pi_{c}(t, \mathbf{x}) \\
& -m \mathcal{P} \tan (m t) \phi(t, \mathbf{x}) .
\end{aligned}
$$

To be as clear as possible, we express $\mathcal{P}$ and $\mathcal{P}^{1 / 2}$ as integral operators,

$$
\begin{aligned}
(\mathcal{P} f)(\mathbf{x}) & \equiv \int d^{3} y \mathcal{P}(\mathbf{x}, \mathbf{y}) f(y), \\
\left(\mathcal{P}^{1 / 2} f\right)(\mathbf{x}) & \equiv \int d^{3} y \mathcal{P}^{1 / 2}(\mathbf{x}, \mathbf{y}) f(y),
\end{aligned}
$$

where $\mathcal{P}(\mathbf{x}, \mathbf{y})$ and $\mathcal{P}^{1 / 2}(\mathbf{x}, \mathbf{y})$ are both symmetric, and $\mathcal{P}^{1 / 2}$ is the operator square root of $\mathcal{P}$, defined by

$$
\int d^{3} z \mathcal{P}^{1 / 2}(\mathbf{x}, \mathbf{z}) \mathcal{P}^{1 / 2}(\mathbf{z}, \mathbf{y})=\mathcal{P}(\mathbf{x}, \mathbf{y}) .
$$

It can then be seen that it is possible to find $F_{2}$ by functionally integrating the first two of Eqs. (A3), where the consistency of the term involving both $\phi$ and $\pi_{c}$ requires that $\mathcal{C}_{1} \mathcal{C}_{2}=2$. We choose $\mathcal{C}_{1}=\mathcal{C}_{2}=\sqrt{2}$, so that

$$
\psi(t, \mathbf{x})=\frac{1}{\sqrt{2}}\left(\psi_{c}(t, \mathbf{x})+i \pi_{c}(t, \mathbf{x})\right),
$$

in agreement with Eq. (18). $F_{2}$ is then given by

$$
\begin{aligned}
F_{2}\left[\phi, \pi_{c}, t\right]= & \sqrt{m} \sec (m t) \int d^{3} x d^{3} y \phi(t, \mathbf{x}) \mathcal{P}^{1 / 2}(\mathbf{x}, \mathbf{y}) \pi_{c}(t, \mathbf{y}) \\
& -\frac{1}{2} m \tan (m t) \int d^{3} x d^{3} y \phi(t, \mathbf{x}) \mathcal{P}(\mathbf{x}, \mathbf{y}) \phi(t, \mathbf{y}) \\
& -\frac{1}{2} \tan (m t) \int d^{3} x \pi_{c}^{2}(t, \mathbf{x}) .
\end{aligned}
$$

To calculate the change in the Hamiltonian from the third of Eqs. (A3), one differentiates Eq. (A9) with respect to $t$ and then replaces $\phi(t, \mathbf{x})$ by using

$$
\left(\mathcal{P}^{1 / 2} \phi\right)(t, \mathbf{x})=\frac{1}{\sqrt{m}}\left[\cos (m t) \psi_{c}(t, \mathbf{x})+\sin (m t) \pi_{c}(t, \mathbf{x})\right],
$$

which follows from Eqs. (A4), along with Eqs. (A1) and (A2). The result is 
$\Delta H=\frac{\partial F_{2}}{\partial t}=-\frac{1}{2} m \int d^{3} x\left\{\psi_{c}^{2}(t, \mathbf{x})+\pi_{c}^{2}(t, \mathbf{x})\right\}$.

To find the new Hamiltonian, we must also express the original Hamiltonian, given by Eq. (2), in terms of the new variables. Note that

$$
\begin{aligned}
\frac{1}{2} \int d^{3} x\left\{m^{2} \phi^{2}+(\nabla \phi)^{2}\right\} & =\frac{1}{2} \int d^{3} x\left\{m^{2} \phi^{2}-\phi \nabla^{2} \phi\right\} \\
& =\frac{m^{2}}{2} \int d^{3} x \phi \mathcal{P}^{2} \phi .
\end{aligned}
$$

It is then straightforward to show that the free part of the original Hamiltonian can be written as

$$
H_{\text {free }}=\frac{1}{2} m \int d^{3} x\left\{\pi_{c} \mathcal{P} \pi_{c}+\psi_{c} \mathcal{P} \psi_{c}\right\} .
$$

The full new Hamiltonian, as prescribed by the third of Eqs. (A3), is then given by

$H_{\text {new }}=\int d^{3} x \mathcal{H}_{\text {new }}$,

$\mathcal{H}_{\text {new }}=m \psi^{*}(\mathcal{P}-1) \psi+\frac{\lambda}{96 m^{2}}\left[e^{-i m t} \psi+e^{i m t} \psi^{*}\right]^{4}$,

where $\psi$ is given by Eq. (A8). Note that this result is in complete agreement with Eq. (19).

\section{APPENDIX B: LOCAL VS NONLOCAL FIELD REDEFINITION}

In order to obtain the effective field theory for the nonrelativistic field we have used the nonlocal field redefinition of Eqs. (7), in contrast to the local redefinition of Eqs. (6), which is more typically found in the literature. As discussed in Secs. II and III, the nonlocal field redefinition makes the computations easier, although it is not fundamentally necessary. In this appendix, we begin with the local field redefinition of Eqs. (6) and obtain the same low-energy effective theory at order $n=1$. As a separate, nontrivial consistency check, we compute the effective field theory for free fields up to order $\epsilon_{x}^{5} \sim(k / m)^{10}$ (in Fourier space) and again find results compatible with those obtained with the nonlocal field redefinition, even though, a priori, the results appear rather different. To demonstrate the equivalence, we remove higher-order timederivative operators that appear in the EFT in favor of spatial operators, using the equations of motion.

\section{Effective field theory through $\boldsymbol{n}=\mathbf{1}$}

We begin with a local field redefinition, in which the relativistic field $\phi$ and the nonrelativistic field $\chi$ are related by

$$
\begin{aligned}
& \phi(t, \mathbf{x})=\frac{1}{\sqrt{2 m}}\left[e^{-i m t} \chi(t, \mathbf{x})+e^{i m t} \chi^{*}(t, \mathbf{x})\right], \\
& \pi(t, \mathbf{x})=-i \sqrt{\frac{m}{2}}\left[e^{-i m t} \chi(t, \mathbf{x})-e^{i m t} \chi^{*}(t, \mathbf{x})\right] .
\end{aligned}
$$

By comparing these equations with Eqs. (7), one can see that $\chi$ is related to the field $\psi$ that we used in the main body of this article by the field redefinition

$\chi=\frac{1}{2}\left(\mathcal{P}^{-1 / 2}+\mathcal{P}^{1 / 2}\right) \psi+\frac{1}{2} e^{2 i m t}\left(\mathcal{P}^{-1 / 2}-\mathcal{P}^{1 / 2}\right) \psi^{*}$,

$\psi=\frac{1}{2}\left(\mathcal{P}^{1 / 2}+\mathcal{P}^{-1 / 2}\right) \chi+\frac{1}{2} e^{2 i m t}\left(\mathcal{P}^{1 / 2}-\mathcal{P}^{-1 / 2}\right) \chi^{*}$.

The equation of motion for $\chi$ takes the form

$i \dot{\chi}(t, \mathbf{x})=-\frac{1}{2 m} \nabla^{2} \chi-\frac{e^{2 i m t}}{2 m} \nabla^{2} \chi^{*}+\frac{\lambda}{4 ! m^{2}} \tilde{G}^{\mathrm{loc}}(t, \mathbf{x})$,

where we have defined

$$
\tilde{G}^{\mathrm{loc}}(t, \mathbf{x}) \equiv e^{i m t}\left[e^{-i m t} \chi+e^{i m t} \chi^{*}\right]^{3},
$$

which matches to the similar object, $\tilde{G}$, defined in Eq. (27), in the limit $\mathcal{P} \rightarrow 1$. Note from Eq. (B3) that the global $U(1)$ symmetry is already broken at the free-field level, obscuring the conservation of particle number in the nonrelativistic limit. As before, we decompose the field into different modes by

$$
\chi(t, \mathbf{x})=\sum_{\nu=-\infty}^{\infty} \chi_{\nu}(t, \mathbf{x}) e^{i \nu m t}
$$

and we define the mode with $\nu=0$ as the slowly varying portion of the field (in whose evolution we are interested),

$$
\chi_{\nu=0}(t, \mathbf{x}) \equiv \chi_{s}(t, \mathbf{x})
$$

We then expand $\tilde{G}^{\text {loc }}$ in a series similar to $\chi$

$$
\tilde{G}^{\mathrm{loc}}(t, \mathbf{x})=\sum_{\nu=-\infty}^{\infty} \tilde{G}_{\nu}^{\mathrm{loc}}(t, \mathbf{x}) e^{i \nu m t}
$$

For a given mode $\nu$, Eq. (B3) then takes the form

$$
i \dot{\chi}_{\nu}-\nu m \chi_{\nu}=-\frac{1}{2 m} \nabla^{2} \chi_{\nu}-\frac{1}{2 m} \nabla^{2} \chi_{2-\nu}^{*}+\frac{\lambda}{4 ! m^{2}} \tilde{G}_{\nu}^{\mathrm{loc}},
$$

with 


$$
\begin{aligned}
\tilde{G}_{\nu}^{\mathrm{loc}}(t, \mathbf{x})= & \sum_{\mu, \mu^{\prime}}\left\{\chi_{\mu} \chi_{\mu^{\prime}} \chi_{2+\nu-\mu-\mu^{\prime}}+\chi_{\mu}^{*} \chi_{\mu^{\prime}}^{*} \chi_{4-\nu-\mu-\mu^{\prime}}^{*}\right. \\
& \left.+3 \chi_{\mu} \chi_{\mu^{\prime}} \chi_{\mu+\mu^{\prime}-\nu}^{*}+3 \chi_{\mu}^{*} \chi_{\mu^{\prime}}^{*} \chi_{\nu-2+\mu+\mu^{\prime}}\right\} .
\end{aligned}
$$

For the slowly varying mode, $\chi_{s}$, Eqs. (B8) and (B9) yield

$$
i \dot{\chi}_{s}=-\frac{1}{2 m} \nabla^{2} \chi_{s}-\frac{1}{2 m} \nabla^{2} \chi_{2}^{*}+\frac{\lambda}{4 ! m^{2}} \tilde{G}_{0}^{\mathrm{loc}},
$$

where

$$
\begin{aligned}
\tilde{G}_{0}^{\mathrm{loc}}= & \sum_{\mu, \mu^{\prime}}\left\{\chi_{\mu} \chi_{\mu^{\prime}} \chi_{2-\mu-\mu^{\prime}}+\chi_{\mu}^{*} \chi_{\mu^{\prime}}^{*} \chi_{4-\mu-\mu^{\prime}}^{*}+3 \chi_{\mu} \chi_{\mu^{\prime}} \chi_{\mu+\mu^{\prime}}^{*}\right. \\
& \left.+3 \chi_{\mu}^{*} \chi_{\mu^{\prime}}^{*} \chi_{\mu+\mu^{\prime}-2}\right\} .
\end{aligned}
$$

Equations (B8) and (B10) reveal that even at linear order in the modes $\chi_{\nu}$, different modes couple to each other. This is one source of complication in the local field redefinition approach. The other difficulty is that powers of the spatial Laplacian appear at each order of the iteration, rather than having them all contained in the operator $\mathcal{P}$. As a result, we must expand in powers of all three small quantities $\left(\epsilon_{t}, \epsilon_{x}\right.$, and $\lambda$ ) from the beginning of our iterative computations. This is in contrast with the calculation in Sec. III, in which we used the nonlocal field redefinition, which enabled us to conduct most of the iterative calculation, until the final step, in terms of just two small parameters $\left(\epsilon_{t}\right.$ and $\lambda$ ). Apart from these technical difficulties, however, nothing prevents us from obtaining an effective field theory for $\chi_{s}$ using Eqs. (B8)-(B11).

It is instructive to rewrite (B8) as

$\nu m \chi_{\nu}=i \dot{\chi}_{\nu}+\frac{1}{2 m} \nabla^{2} \chi_{\nu}+\frac{1}{2 m} \nabla^{2} \chi_{2-\nu}^{*}-\frac{\lambda}{4 ! m^{2}} \tilde{G}_{\nu}^{\mathrm{loc}}$,

where, in contrast with the nonlocal case in Eq. (32), we have kept the prefactors on the left-hand side to avoid the apparent divergence for $\nu=0$. The right-hand side of Eq. (B12) can be thought of as a perturbative source for $\chi_{\nu}$ : the first term is suppressed relative to $\chi_{\nu}$ by $\epsilon_{t}$, the second and third terms are suppressed relative to $\chi_{\nu}$ and $\chi_{2-\nu}$ by $\epsilon_{x}$, and the last term is suppressed relative to $\tilde{G}_{\nu}^{\text {loc }}$ by $\lambda$. Therefore, as noted above, our expansion in this case is in powers of all three small quantities.

We next expand the modes with $\nu \neq 0$ :

$$
\chi_{\nu}(t, \mathbf{x})=\sum_{n=1}^{\infty} \chi_{\nu}^{(n)}(t, \mathbf{x}) \text { for } \nu \neq 0,
$$

where the terms $\chi_{\nu}^{(n)}$ are to be determined by iterative approximation, as described below. We also expand $\tilde{G}_{\nu}^{\text {loc }}$ :

$$
\tilde{G}_{\nu}^{\mathrm{loc}}(t, \mathbf{x})=\sum_{n=0}^{\infty} \tilde{G}_{\nu}^{\mathrm{loc}^{(n)}}(t, \mathbf{x})
$$

where $\tilde{G}_{\nu}^{\text {loc }}{ }^{(n)}(t, \mathbf{x})$ consists of all terms in Eq. (B11) which are proportional to a total of $n$ powers of the small quantities $\epsilon_{t}, \epsilon_{x}$, and $\lambda$. Note that we decompose $\chi_{\nu}$ only for $\nu \neq 0$, and, as in Sec. III, we set $\chi_{0}^{(0)} \equiv \chi_{s}, \chi_{0}^{(n)}=0$ for $n>0$, and $\chi_{\nu}^{(0)}=0$ for $\nu \neq 0$. Expanding (B12) yields

$$
\begin{aligned}
\nu m \chi_{\nu}^{(1)}= & \frac{1}{2 m} \nabla^{2} \chi_{s}^{*} \delta_{2, \nu}-\frac{\lambda}{4 ! m^{2}} \tilde{G}_{\nu}^{\mathrm{loc}^{(0)}} \quad \text { for } \nu \neq 0, \\
\nu m \chi_{\nu}^{(n)}= & i \dot{\chi}_{\nu}^{(n-1)}+\frac{1}{2 m} \nabla^{2} \chi_{\nu}^{(n-1)}+\frac{1}{2 m} \nabla^{2} \chi_{2-\nu}^{(n-1) *} \\
& -\frac{\lambda}{4 ! m^{2}} \tilde{G}_{\nu}^{\mathrm{loc}^{(n-1)}} \text { for } \nu \neq 0 \text { and } n \geq 2 .
\end{aligned}
$$

From this iterative expansion one can obtain the effective equation for $\chi_{s}$ :

$i \dot{\chi}_{s}=-\frac{1}{2 m} \nabla^{2} \chi_{s}-\frac{1}{2 m} \nabla^{2} \sum_{n=1}^{\infty} \chi_{2}^{(n) *}+\frac{\lambda}{4 ! m^{2}} \sum_{n=0}^{\infty} \tilde{G}_{0}^{\mathrm{loc}^{(n)}}$.

As in Sec. III, we only compute the leading-order corrections to the Schrödinger equation for $\chi_{s}$. From Eq. (B9), we have

$$
\tilde{G}_{\nu}^{\mathrm{loc}(0)}=\chi_{s}^{3} \delta_{\nu,-2}+\chi_{s}^{* 3} \delta_{\nu, 4}+3\left|\chi_{s}\right|^{2} \chi_{s}^{*} \delta_{\nu, 2}+3\left|\chi_{s}\right|^{2} \chi_{s} \delta_{\nu, 0},
$$

and then to order $n=1$, we find

$$
\begin{aligned}
\chi_{\nu}^{(1)}= & \frac{1}{4 m^{2}} \nabla^{2} \chi_{s}^{*} \delta_{\nu, 2}+\frac{\lambda}{2 \times 4 ! m^{3}}\left[\chi_{s}^{3} \delta_{\nu,-2}-\frac{1}{2} \chi_{s}^{* 3} \delta_{\nu, 4}\right. \\
& \left.-3\left|\chi_{s}\right|^{2} \chi_{s}^{*} \delta_{\nu, 2}\right] \text { for } \nu \neq 0 .
\end{aligned}
$$

Again from Eq. (B9), we find

$$
\begin{aligned}
\tilde{G}_{\nu}^{\text {loc }}{ }^{(1)}= & 3\left[\chi_{s}^{2} \chi_{2+\nu}^{(1)}+\chi_{s}^{* 2} \chi_{4-\nu}^{(1) *}+\chi_{s}^{2} \chi_{-\nu}^{(1) *}+2\left|\chi_{s}\right|^{2} \chi_{\nu}^{(1)}\right. \\
& \left.+\chi_{s}^{* 2} \chi_{\nu-2}^{(1)}+2\left|\chi_{s}\right|^{2} \chi_{2-\nu}^{(1) *}\right],
\end{aligned}
$$

so

$$
\begin{aligned}
i \dot{\chi}_{s}= & -\frac{1}{2 m} \nabla^{2} \chi_{s}+\frac{3 \lambda}{4 ! m^{2}}\left|\chi_{s}\right|^{2} \chi_{s}-\frac{1}{2 m} \nabla^{2} \chi_{2}^{(1) *} \\
& +\frac{3 \lambda}{4 ! m^{2}}\left[\chi_{s}^{2} \chi_{2}^{(1)}+\chi_{s}^{* 2} \chi_{4}^{(1) *}+\chi_{s}^{* 2} \chi_{-2}^{(1)}+2\left|\chi_{s}\right|^{2} \chi_{2}^{(1) *}\right] \\
& +\mathcal{O}\left(\epsilon^{2}\right) .
\end{aligned}
$$

For convenience, we have introduced the shorthand notation

$$
\begin{aligned}
& \mathcal{O}\left(\epsilon^{2}\right) \equiv \mathcal{O}\left[\epsilon_{x}^{2}, \epsilon_{t}^{2}, \lambda^{2}, \epsilon_{x} \epsilon_{t}, \epsilon_{x} \lambda, \epsilon_{t} \lambda\right] \\
& \mathcal{O}\left(\epsilon^{3}\right) \equiv \mathcal{O}\left[\epsilon_{x}^{3}, \epsilon_{t}^{3}, \lambda^{3}, \epsilon_{x}^{2} \epsilon_{t}, \epsilon_{x}^{2} \lambda, \epsilon_{x} \epsilon_{t}^{2}, \epsilon_{x} \lambda^{2}, \epsilon_{x} \epsilon_{t} \lambda, \epsilon_{t}^{2} \lambda, \epsilon_{t} \lambda^{2}\right]
\end{aligned}
$$


Next we substitute $\chi_{\nu}^{(1)}$ from Eq. (B18) into Eq. (B20), which yields

$$
\begin{aligned}
i \dot{\chi}_{s} \simeq & -\frac{1}{2 m} \nabla^{2} \chi_{s}+\frac{\lambda}{8 m^{2}}\left|\chi_{s}\right|^{2} \chi_{s}-\frac{1}{8 m^{3}} \nabla^{4} \chi_{s} \\
& +\frac{\lambda}{32 m^{4}}\left[\chi_{s}^{2} \nabla^{2} \chi_{s}^{*}+2\left|\chi_{s}\right|^{2} \nabla^{2} \chi_{s}+\nabla^{2}\left(\left|\chi_{s}\right|^{2} \chi_{s}\right)\right] \\
& -\frac{17 \lambda^{2}}{768 m^{5}}\left|\chi_{s}\right|^{4} \chi_{s}+\mathcal{O}\left(\epsilon^{3}\right) .
\end{aligned}
$$

Equation (B22) for $\chi_{s}$ exactly matches Eq. (43) for $\psi_{s}$, which is related to the relativistic field $\phi$ via the nonlocal field redefinition of Eqs. (7).

Since $\chi$ and $\psi$ are related to each other by the nontrivial field redefinition of Eqs. (B2), there was no guarantee that $\chi_{s}$ and $\psi_{s}$ should obey the same equation. The two field theories must be equivalent, since they are both equivalent to the low-energy effective field theory derived from the relativistic $\phi^{4}$ theory, but $\chi_{s}$ and $\psi_{s}$ could be related by a field redefinition that causes them to have different equations of motion. We have found, however, that through $\mathcal{O}\left(\epsilon^{2}\right)$, the equations for $\chi_{s}$ and $\psi_{s}$ are identical. We do not know if this relation will continue to hold at higher orders. However, for the special case of the free field theory, $\lambda=0$, the relation between $\chi_{s}$ and $\psi_{s}$ is simple. For the free field theory, $\psi$ itself is slowly varying, so $\psi_{s}=\psi$. Then in the Eq. (B2a), which expresses $\chi$ in terms of $\psi$, the first term is purely slowly varying, while the second term is the product of $e^{2 i m t}$ and a slowly varying function. Thus, the first term is the slowly varying part of $\chi$ :

$$
\chi_{s}=\frac{1}{2}\left(\mathcal{P}^{-1 / 2}+\mathcal{P}^{1 / 2}\right) \psi_{s} .
$$

Since $\left(\mathcal{P}^{-1 / 2}+\mathcal{P}^{1 / 2}\right)$ commutes with the differential operators in the equation of motion, $\chi_{s}$ obeys the same equation of motion as $\phi_{s}$,

$$
i \dot{\chi}_{s}=m(\mathcal{P}-1) \chi_{s} .
$$

The iterative procedure used above can be continued to obtain higher- and higher-order corrections to the Schrödinger equation. Generically, higher-order terms contain higher-order time-derivative operators, as expected in an EFT framework, and as was discussed after Eq. (43) and at the end of Sec. III. The higher-order time-derivative terms do not introduce new d.o.f., because such terms must be considered perturbations around the zeroth-order equations. In fact, one can use the equations of motion to remove the higher-order time-derivative terms and replace them with lower-order terms [25]. (See also [26,27].) We demonstrate this explicitly in the following subsection for $\chi$ defined via the local field redefinition of Eq. (B1), in the free-field limit (taking $\lambda \rightarrow 0$ ). This limit is sufficiently nontrivial that a generalization to the interacting case would not be straightforward. We also show that the final result, after removing the higher-order time derivatives, is of exactly the form one would obtain for $\psi$, as defined via the nonlocal field redefinition. We pursue the comparison up to order $\epsilon_{x}^{5} \sim(k / m)^{10}$.

\section{Higher-order time-derivative terms in the free-field limit}

In the free-field limit the equation of motion for $\chi$, Eq. (B3), simplifies to

$$
i \dot{\chi}=-\frac{1}{2 m} \nabla^{2} \chi-\frac{e^{2 i m t}}{2 m} \nabla^{2} \chi^{*} .
$$

Equation (B25) (together with its complex conjugate) can be solved exactly, with a solution of the form of Eq. (B2a). As discussed above, the solution is the sum of two terms, one that varies slowly, and the other that varies rapidly. Thus it is straightforward to write an exact expression for the slow mode, Eq. (B23). However, here we will put aside the exact solution, and use this case to further illustrate the iterative approximation technique, as was used in Appendix B 1.

After decomposing the field $\chi$ into modes as in Eq. (B5) and again assigning $\chi_{\nu=0} \equiv \chi_{s}$, the equation of motion for $\chi_{s}$ takes the form

$$
i \dot{\chi}_{s}=-\frac{1}{2 m} \nabla^{2} \chi_{s}-\frac{1}{2 m} \nabla^{2} \chi_{2}^{*} .
$$

Therefore, among all modes $\chi_{\nu}$ with $\nu \neq 0$, only $\chi_{2}$ contributes to the evolution of $\chi_{s}$ in the limit $\lambda \rightarrow 0$. So we only need to find an appropriate substitution for $\chi_{2}$ in order to obtain the EFT for $\chi_{s}$ in this limit. From Eq. (B15) we may write

$$
i \dot{\chi}_{2}-2 m \chi_{2}=-\frac{1}{2 m} \nabla^{2} \chi_{2}-\frac{1}{2 m} \nabla^{2} \chi_{s}^{*} .
$$

Decomposing $\chi_{2}$ as in Eq. (B13) it is easy to obtain

$$
\chi_{2}^{(1)}=\frac{1}{4 m^{2}} \nabla^{2} \chi_{s}^{*},
$$

while for higher-order iterations we have

$$
\chi_{2}^{(n)}=\frac{1}{2 m}\left(\frac{1}{2 m} \nabla^{2}+i \partial_{t}\right) \chi_{2}^{(n-1)}, \quad \text { for } n \geq 2
$$

all of which can be encapsulated in the relation

$$
\chi_{2}^{(n)}=\frac{1}{4 m^{2}} \nabla^{2}\left(\frac{1}{4 m^{2}} \nabla^{2}+\frac{i}{2 m} \partial_{t}\right)^{n-1} \chi_{s}^{*} .
$$

Having obtained $\chi_{2}$ at arbitrary iteration $n$, one may try to formally resum the infinite series to obtain 
$\chi_{2}=\sum_{n=1}^{\infty} \chi_{2}^{(n)}=\frac{1}{4 m^{2}} \nabla^{2}\left(1-\frac{1}{4 m^{2}} \nabla^{2}-\frac{i}{2 m} \partial_{t}\right)^{-1} \chi_{s}^{*}$.

Substituting this relation back to Eq. (B26) yields

$$
i \dot{\chi}_{s}=-\frac{1}{2 m} \nabla^{2} \chi_{s}-\frac{1}{8 m^{3}} \nabla^{4}\left(1-\frac{1}{4 m^{2}} \nabla^{2}+\frac{i}{2 m} \partial_{t}\right)^{-1} \chi_{s} .
$$

Although the infinite series has been resummed, one can check that the exact solution of Eq. (B26) would satisfy Eq. (B32). Here we will only consider the first few terms among the infinite tower of terms in order to demonstrate how higher-order time derivatives enter the calculation and may be systematically removed. To do so, it is easiest to work in Fourier space, within which we expand Eq. (B32) up to the order $(k / m)^{10}$ :

$$
\begin{aligned}
i \dot{\chi}_{s} \simeq & \frac{k^{2}}{2 m} \chi_{s} \\
& -\frac{k^{4}}{8 m^{3}} \chi_{s} \\
& +\frac{i k^{4}}{16 m^{4}} \dot{\chi}_{s}+\frac{k^{6}}{32 m^{5}} \chi_{s} \\
& +\frac{k^{4}}{32 m^{5}} \ddot{\chi}_{s}-\frac{i k^{6}}{32 m^{6}} \dot{\chi}_{s}-\frac{k^{8}}{2^{7} m^{7}} \chi_{s} \\
& -\frac{i k^{4}}{64 m^{6}} \ddot{\chi}_{s}-\frac{3 k^{6}}{2^{7} m^{7}} \ddot{\chi}_{s}+\frac{3 i k^{8}}{2^{8} m^{8}} \dot{\chi}_{s}+\frac{k^{10}}{2^{9} m^{9}} \chi_{s} \\
& +\mathcal{O}\left[(k / m)^{12}\right] .
\end{aligned}
$$

In Eq. (B33) we have arranged terms of similar magnitude to be in the same line. Notice that from the equation of motion, Eq. (B26), one can conclude that the timederivative operator and the spatial Laplacian are at the same order (i.e., in Fourier space, $\partial_{t} \sim k^{2} / m$ ) so that, effectively, our expansion is in powers of $(\mathrm{k} / \mathrm{m})$.

Equation (B33) is a higher-order equation in time derivatives. But, as mentioned above, since all terms from the second to the fifth line should be considered as perturbations, we can replace higher-order time-derivative terms with lower-order terms, upon using the equation of motion and its time derivatives. The procedure is simple. First, consider Eq. (B33) as an algebraic equation for $\dot{\chi}_{s}$; move all terms involving $\dot{\chi}_{s}$ to the left-hand side; divide both sides by the resulting coefficient:

$$
\left(1-\frac{k^{4}}{16 m^{4}}+\frac{k^{6}}{32 m^{6}}-\frac{3 k^{8}}{2^{8} m^{8}}\right)
$$

and expand up to the desired order (here $\left.\mathcal{O}(k / m)^{10}\right)$. This yields

$$
\begin{aligned}
i \dot{\chi}_{s} \simeq & \frac{k^{2}}{2 m} \chi_{s}-\frac{k^{4}}{8 m^{3}} \chi_{s}+\frac{k^{6}}{16 m^{5}} \chi_{s}+\frac{k^{4}}{32 m^{5}} \ddot{\chi}_{s}-\frac{k^{8}}{32 m^{7}} \chi_{s} \\
& -\frac{i k^{4}}{64 m^{6}} \dddot{\chi}_{s}-\frac{3 k^{6}}{2^{7} m^{7}} \ddot{\chi}_{s}+\frac{k^{10}}{64 m^{9}} \chi_{s}+\mathcal{O}\left[(k / m)^{12}\right] .
\end{aligned}
$$

The right-hand side of Eq. (B35) contains no first timederivative terms, though it still contains higher-order ones. To remove the $\ddot{\chi}_{s}$ terms, we take a time derivative of Eq. (B35) once and remove terms containing $\dot{\chi}_{s}$ using Eq. (B35) itself, again only keeping terms up to the desired order in $(\mathrm{k} / \mathrm{m})$. This yields an algebraic equation for $\ddot{\chi}_{s}$ that does not contain $\dot{\chi}_{s}$. Using the resulting equation to remove $\ddot{\chi}_{s}$ from Eq. (B35) yields

$$
\begin{aligned}
i \dot{\chi}_{s} \simeq & \frac{k^{2}}{2 m} \chi_{s}-\frac{k^{4}}{8 m^{3}} \chi_{s}+\frac{k^{6}}{16 m^{5}} \chi_{s}-\frac{5 k^{8}}{2^{7} m^{7}} \chi_{s} \\
& -\frac{i k^{4}}{64 m^{6}} \dddot{\chi}_{s}+\frac{13 k^{10}}{2^{9} m^{9}} \chi_{s} .
\end{aligned}
$$

By this series of steps, we have removed $\ddot{\chi}_{s}$ from the equation. We repeat the same procedure to remove $\dddot{\chi}_{s}$ by taking the time derivative of Eq. (B36) twice and substituting the resulting expression for $\dddot{\chi}_{s}$ into Eq. (B36). This finally yields

$$
\begin{aligned}
i \dot{\chi}_{s}= & {\left[\frac{k^{2}}{2 m}-\frac{k^{4}}{8 m^{3}}+\frac{k^{6}}{16 m^{4}}-\frac{5 k^{8}}{128 m^{7}}+\frac{7 k^{10}}{256 m^{9}}\right] \chi_{s} } \\
& +\mathcal{O}\left[(k / m)^{12}\right],
\end{aligned}
$$

which no longer includes any higher-order time-derivative terms. The form of Eq. (B37) is in complete agreement (up to the working order) with the expansion of the equation of motion in the free-field limit that one would obtain by using the nonlocal field redefinition, namely

$$
i \dot{\psi}_{s}=m\left(\sqrt{1+\frac{k^{2}}{m^{2}}}-1\right) \psi_{s}
$$

which is the Fourier transform of Eq. (40) in the limit $\lambda \rightarrow 0$.

\section{APPENDIX C: COMPARISON WITH THE RECENT CALCULATION BY MUKAIDA, TAKIMOTO, AND YAMADA}

In this Appendix we compare our EFT with the recent calculation by Mukaida, Takimoto, and Yamada in Ref. [11]. We show that the two resulting low-energy descriptions are equivalent, related by a field redefinition.

In Ref. [11] the authors begin by separating the real-valued scalar field of the relativistic theory, $\phi$, into a nonrelativistic 
component and fluctuations, $\phi(x)=\phi_{\mathrm{NR}}(x)+\delta \phi(x)$. The nonrelativistic field $\phi_{\mathrm{NR}}$ is defined as

$$
\phi_{\mathrm{NR}}(x)=\int_{K \in \mathrm{NR}} d^{4} K e^{-i K \cdot x} \phi(K),
$$

where $K \in \mathrm{NR}$ indicates the region of four-momentum $K^{\mu}=\left(k^{0}, \mathbf{k}\right)$ with $\left|k^{0}\right| \sim m c^{2}+\mathcal{O}\left(m v^{2}\right)$ and $0 \leq|\mathbf{k}| \leq m v$, with $v \ll c$. (The fluctuation $\delta \phi$ is defined as the Fourier integral over the complementary range of $K^{\mu}$.) Next the authors parameterize the nonrelativistic component as

$$
\phi_{\mathrm{NR}}(t, \mathbf{x})=\frac{1}{2}\left[\sigma(t, \mathbf{x}) e^{-i m t}+\sigma^{*}(t, \mathbf{x}) e^{i m t}\right] .
$$

(The field we have labeled $\sigma$ is denoted by $\Psi$ in Ref. [11]; we use $\sigma$ to avoid confusion with the modes $\Psi_{\nu}$ defined in Eq. (31).) In contrast with our approach, the authors of Ref. [11] first separate the relativistic field $\phi$ into components with small and large spatial momenta, $\sigma$ and $\delta \phi$ (respectively), and then construct an EFT for $\sigma$, whereas we relate the realvalued relativistic field $\phi$ to the complex field $\psi$ via Eqs. (7) and construct an EFT for the slowly varying portion, $\psi_{s}$, identified as the $\nu=0$ mode of Eq. (24). This suggests that the two resulting low-energy effective descriptions might be related by a field redefinition. In particular, substituting the mode decomposition of Eq. (24) into Eq. (7), we may relate $\sigma$ to $\psi$ :

$$
\begin{aligned}
\frac{1}{2} & {\left[\sigma(t, \mathbf{x}) e^{-i m t}+\sigma^{*}(t, \mathbf{x}) e^{i m t}\right] } \\
& =\frac{1}{\sqrt{2 m}} \mathcal{P}^{-1 / 2}\left[\left(\psi_{s}+\psi_{2}^{*}\right) e^{-i m t}+\left(\psi_{s}^{*}+\psi_{2}\right) e^{i m t}\right] .
\end{aligned}
$$

Since $\sigma, \psi_{s}$, and $\psi_{2}$ are each slowly varying functions of $t$, Eq. (C3) implies that

$$
\sqrt{\frac{m}{2}} \sigma=\mathcal{P}^{-1 / 2}\left(\psi_{s}+\psi_{2}^{*}\right) .
$$

At leading order in $\epsilon_{x}, \epsilon_{t}$, and $\lambda$, this reduces to

$$
\sqrt{\frac{m}{2}} \sigma=\left(1+\frac{1}{4 m^{2}} \nabla^{2}\right) \psi_{s}-\frac{\lambda}{16 m^{3}}\left|\psi_{s}\right|^{2} \psi_{s}+\mathcal{O}\left(\epsilon^{2}\right),
$$

where we have expanded the nonlocal operator $\mathcal{P}$, used the relations in Eqs. (38) and (41) to replace $\psi_{2}^{*}$, and adopted the notation $\mathcal{O}\left(\epsilon^{2}\right)$ of Eq. (B21) to indicate terms that are at least second order in the small quantities $\epsilon_{x}, \epsilon_{t}$, and $\lambda$.

Before showing the equivalence of the low-energy effective descriptions for $\sigma$ and $\psi_{s}$, we note that one can derive the same relation as in Eq. (C5), starting from the locally defined field $\chi$ of Eq. (B1). In that case, the relation between the two fields becomes $\sqrt{m / 2} \sigma=\chi_{s}+\chi_{2}^{*}$, which differs from Eq. (C4) by the absence of the nonlocal operator $\mathcal{P}$. However, according to Eq. (B18), the $\chi_{2}^{*}$ term acquires an extra contribution that exactly compensates the missing term due to the absence of $\mathcal{P}$ from the relation between $\sigma$ and $\chi$.

To demonstrate that the low-energy effective descriptions for $\sigma$ and $\psi_{s}$ are indeed related through the field redefinition of Eq. (C5), we first consider the equations of motion. In Eq. (2.15) of Ref. [11] the authors present their effective Lagrangian, which is given by

$$
\mathcal{L}=\frac{1}{4}\left[2 i m \sigma^{*} \dot{\sigma}-\sigma^{*} \ddot{\sigma}+\sigma^{*} \nabla^{2} \sigma-V_{\text {eff }}\right],
$$

where, from Eq. (2.17) of Ref. [11],

$$
V_{\text {eff }}\left(\sigma, \sigma^{*}\right)=-\frac{3 g_{4}}{8}|\sigma|^{4}+\frac{g_{4}^{2}}{128 m^{2}}|\sigma|^{6} .
$$

Here $g_{4}$ is the coupling constant of the quartic interaction in the original, relativistic theory, and we have set the cubic interaction to zero, to match the form of $V(\phi)$ that we have considered throughout our analysis. The quartic coupling constant $g_{4}$ of Ref. [11] is related to the coupling $\lambda$ we introduced in Eq. (1) by $g_{4}=-\lambda / 6$.

The equation of motion for $\sigma$, up to the order that matches our analysis, can be obtained by varying the action with respect to $\sigma^{*}$, which yields

$$
i \dot{\sigma}=\frac{1}{2 m} \ddot{\sigma}-\frac{1}{2 m} \nabla^{2} \sigma+\frac{1}{2 m} V_{\mathrm{eff}, \sigma^{*}}
$$

Equation (C8) is second order in time derivatives. Upon substituting the field redefinition of Eq. (C5), we find an equation of motion for $\psi_{s}$ which is also second order in time derivatives:

$$
\begin{aligned}
i \dot{\psi}_{s}= & -\frac{1}{2 m} \nabla^{2} \psi_{s}+\frac{\lambda}{8 m^{2}}\left|\psi_{s}\right|^{2} \psi_{s} \\
& +\frac{1}{2 m} \ddot{\psi}_{s}-\frac{i}{4 m^{2}} \nabla^{2} \dot{\psi}_{s}+\frac{i \lambda}{16 m^{3}} \psi_{s}\left(2 \dot{\psi}_{s} \psi_{s}^{*}+\psi_{s} \dot{\psi}_{s}^{*}\right) \\
& -\frac{1}{8 m^{3}} \nabla^{4} \psi_{s}+\frac{\lambda}{32 m^{4}}\left[\psi_{s}^{2} \nabla^{2} \psi_{s}^{*}+2\left|\psi_{s}\right|^{2} \nabla^{2} \psi_{s}\right. \\
& \left.+\nabla^{2}\left(\left|\psi_{s}\right|^{2} \psi_{s}\right)\right]-\frac{17 \lambda^{2}}{768 m^{5}}\left|\psi_{s}\right|^{4} \psi_{s}+\mathcal{O}\left(\epsilon^{3}\right) .
\end{aligned}
$$

Equation (C9) does not appear to match the equation of motion for $\psi_{s}$ which we found above, in Eq. (43). In particular, the two equations differ by the presence of the terms in the second line of Eq. (C9). However, to the perturbative order to which we are working, it is straightforward to show that the new terms that appear in Eq. (C9) vanish. In particular, we may take one time derivative of Eq. (C9) to find 


$$
i \ddot{\psi}_{s}=-\frac{1}{2 m} \nabla^{2} \dot{\psi}_{s}+\frac{\lambda}{8 m^{2}} \psi_{s}\left(2 \dot{\psi}_{s} \psi_{s}^{*}+\psi_{s} \dot{\psi}_{s}^{*}\right)+\mathcal{O}\left(\epsilon^{3}\right) .
$$

Substituting Eq. (C10) into Eq. (C9) yields

$$
\begin{aligned}
i \dot{\psi}_{s}= & -\frac{1}{2 m} \nabla^{2} \psi_{s}+\frac{\lambda}{8 m^{2}}\left|\psi_{s}\right|^{2} \psi_{s}-\frac{1}{8 m^{3}} \nabla^{4} \psi_{s} \\
& +\frac{\lambda}{32 m^{4}}\left[\psi_{s}^{2} \nabla^{2} \psi_{s}^{*}+2\left|\psi_{s}\right|^{2} \nabla^{2} \psi_{s}+\nabla^{2}\left(\left|\psi_{s}\right|^{2} \psi_{s}\right)\right] \\
& -\frac{17 \lambda^{2}}{768 m^{5}}\left|\psi_{s}\right|^{4} \psi_{s}+\mathcal{O}\left(\epsilon^{3}\right) .
\end{aligned}
$$

To the order to which we have been working, Eq. (C11) exactly matches Eq. (43). We therefore find that the equations of motion for the two low-energy effective descriptions are indeed equivalent, at least up to the working order.

So far our discussion has established the equivalence between the two low-energy descriptions at the classical level. To analyze the equivalence even for matters concerning quantization, we next consider the relevant Lagrangians. Substituting the field redefinition of Eq. (C5) into the Lagrangian of Eq. (C6) and performing some straightforward algebra, we find

$$
\begin{aligned}
\mathcal{L}\left[\psi_{s}\right]= & \frac{i}{2}\left(\dot{\psi}_{s} \psi_{s}^{*}-\psi_{s} \dot{\psi}_{s}^{*}\right)-\frac{1}{2 m} \nabla \psi_{s} \nabla \psi_{s}^{*}-\frac{\lambda}{16 m^{2}}\left|\psi_{s}\right|^{4} \\
& -\frac{1}{2 m} \psi_{s}^{*} \ddot{\psi}_{s}-\frac{i}{2 m^{2}} \dot{\psi}_{s}^{*} \nabla^{2} \psi_{s}+\frac{1}{4 m^{3}} \nabla^{2} \psi_{s} \nabla^{2} \psi_{s}^{*} \\
& -\frac{i \lambda}{8 m^{3}}\left|\psi_{s}\right|^{2} \psi_{s}^{*} \dot{\psi}_{s}-\frac{\lambda}{16 m^{4}}\left|\psi_{s}\right|^{2}\left(\psi_{s}^{*} \nabla^{2} \psi_{s}+\psi_{s} \nabla^{2} \psi_{s}^{*}\right) \\
& +\frac{35 \lambda^{2}}{9 \times 2^{8} m^{5}}\left|\psi_{s}\right|^{6},
\end{aligned}
$$

where we have neglected some boundary terms. The Lagrangian of Eq. (C12) is different from the one we obtained in Eq. (44), although it is easy to show that Eq. (C12) gives rise to the same equations of motion. To demonstrate the equivalence between the Lagrangians in Eqs. (44) and (C12), we perform another field redefinition:

$$
\psi_{s}=\tilde{\psi}-\frac{1}{4 m}\left(i \dot{\tilde{\psi}}+\frac{1}{2 m} \nabla^{2} \tilde{\psi}-\frac{\lambda}{8 m^{2}}|\tilde{\psi}|^{2} \tilde{\psi}\right) .
$$

Substituting Eq. (C13) into Eq. (C12) yields

$$
\begin{aligned}
\mathcal{L}[\tilde{\psi}]= & \frac{i}{2}\left(\dot{\tilde{\psi}} \tilde{\psi}^{*}-\tilde{\psi} \tilde{\tilde{\psi}}^{*}\right)-\frac{1}{2 m} \nabla \tilde{\psi} \nabla \tilde{\psi}^{*}-\frac{\lambda}{16 m^{2}}|\tilde{\psi}|^{4} \\
& +\frac{1}{8 m^{3}} \nabla^{2} \tilde{\psi} \nabla^{2} \tilde{\psi}^{*}-\frac{\lambda}{32 m^{4}}|\tilde{\psi}|^{2}\left(\tilde{\psi}^{*} \nabla^{2} \tilde{\psi}+\tilde{\psi} \nabla^{2} \tilde{\psi}^{*}\right) \\
& +\frac{17 \lambda^{2}}{9 \times 2^{8} m^{5}}|\tilde{\psi}|^{6} .
\end{aligned}
$$

This is precisely the form of the Lagrangian of Eq. (44), after trivial relabeling of the dynamical field $\psi_{s} \rightarrow \tilde{\psi}$. Since the Lagrangians of Eqs. (C6), (C12), and (C14) are related to each other via field redefinitions, we expect their corresponding $S$-matrices to remain equivalent as well, at least up to the perturbative order to which we have been working [25-27].

We close with some comments on the field redefinitions in Eqs. (C5) and (C13). Both of the field redefinitions may be written in the form $\psi \rightarrow \psi+\epsilon T(\psi)$, where $T(\psi)$ is a local function of $\psi$ and its derivatives. As demonstrated by Arzt in Ref. [27] (see also Refs. [25,26]), within an EFT context, such redefinitions do not change the $S$-matrix. We therefore conclude that the low-energy effective description we have derived for the field $\psi_{s}$ is equivalent, to our working order in perturbation theory, to the low-energy description derived in Ref. [11], at both the classical and quantum levels [39].
[1] P. J. E. Peebles, Dark matter, Proc. Nat. Acad. Sci. (USA) 112, 12246 (2015).

[2] J. Preskill, M. B. Wise, and F. Wilczek, Cosmology of the invisible axion, Phys. Lett. 120B, 127 (1983).

[3] L. F. Abbott and P. Sikivie, A cosmological bound on the invisible axion, Phys. Lett. 120B, 133 (1983).

[4] M. Dine and W. Fischler, The not so harmless axion, Phys. Lett. 120B, 137 (1983).

[5] J. E. Kim and G. Carosi, Axions and the strong $C P$ problem, Rev. Mod. Phys. 82, 557 (2010).

[6] A. V. Manohar and I. W. Stewart, Nonrelativistic bound states in quantum field theory, Nucl. Phys. B, Proc. Suppl. 94, 130 (2001).
[7] A. V. Manohar and I. W. Stewart, The QCD heavy-quark potential to order $v^{2}$ : One loop matching conditions, Phys. Rev. D 62, 074015 (2000).

[8] N. Brambilla, A. Pineda, J. Soto, and A. Vairo, Effective field theories for heavy quarkonium, Rev. Mod. Phys. 77, 1423 (2005).

[9] M. Gleiser, Pseudo-stable bubbles, Phys. Rev. D 49, 2978 (1994); E. J. Copeland, M. Gleiser, and H.-J. Mueller, Oscillons: Resonant configurations during bubble collapse, Phys. Rev. D 52, 1920 (1995).

[10] M. A. Amin, R. Easther, and H. Finkel, Inflaton fragmentation and oscillon formation in three dimensions, J. Cosmol. Astropart. Phys. 12 (2010) 001; M. A. Amin, 
R. Easther, H. Finkel, R. Flauger, and M. P. Hertzberg, Oscillons after Inflation, Phys. Rev. Lett. 108, 241302 (2012).

[11] K. Mukaida, M. Takimoto, and M. Yamada, On longevity of I-ball/oscillon, J. High Energy Phys. 03 (2017) 122.

[12] M. Gross and S. Haroche, Superradiance: An essay on the theory of collective spontaneous emission, Phys. Rep. 93, 301 (1982).

[13] A. Arvanitaki and S. Dubovsky, Exploring the string axiverse with precision black hole physics, Phys. Rev. D 83, 044026 (2011).

[14] R. D. Peccei and H. R. Quinn, $C P$ Conservation in the Presence of Instantons, Phys. Rev. Lett. 38, 1440 (1977).

[15] S. Weinberg, A New Light Boson?, Phys. Rev. Lett. 40, 223 (1978).

[16] F. Wilczek, Problem of Strong P and T Invariance in the Presence of Instantons, Phys. Rev. Lett. 40, 279 (1978).

[17] A. H. Guth, M. P. Hertzberg, and C. Prescod-Weinstein, Do dark matter axions form a condensate with long-range correlation?, Phys. Rev. D 92, 103513 (2015).

[18] A. G. Siapas and M. A. Wilson, Coordinated interactions between hippocampal ripples and cortical spindles during slow-wave sleep, Neuron 21, 1123 (1998).

[19] Z. Clemens, M. Möll, L. Eröss, R. Jakus, G. Rásonyi, P. Halász, and J. Born, Fine-tuned coupling between human parahippocampal ripples and sleep spindles, Eur. J. Neurosci. 33, 511 (2011).

[20] E. Braaten, A. Mohapatra, and H. Zhang, Nonrelativistic effective field theory for axions, Phys. Rev. D 94, 076004 (2016).

[21] R. Banerjee and P. Mukherjee, On the subtelties of nonrelativistic reduction and applications, arXiv:1801.08373.

[22] Axion dark matter is expected to be virialized in galaxies, though in that case the dominant contribution to the potential energy arises from gravitational self-interactions rather than from the $\lambda \phi^{4}$ interaction that we study here. See, e.g., Refs. [23,24].

[23] F. Zwicky, Die Rotverschiebung von extragalaktischen Nebeln, Helv. Phys. Acta 6, 110 (1933); F. Zwicky, On the masses of nebulae and of clusters of nebulae, Astrophys. J. 86, 217 (1937).

[24] J. Binney and S. Tremaine, Galactic Dynamics, 2nd ed. (Princeton University Press, Princeton, NJ, 2008), Chap. 9.

[25] C. Grosse-Knetter, Effective Lagrangians and higher order derivatives, Phys. Rev. D 49, 6709 (1994).

[26] H. Georgi, On-shell effective field theory, Nucl. Phys. B361, 339 (1991).

[27] C. Arzt, Reduced effective Lagrangians, Phys. Lett. B 342, 189 (1995).

[28] E. Braaten, A. Mohapatra, and H. Zhang, Emission of photons and relativistic axions from axion stars, Phys. Rev. D 96, 031901(R) (2017).

[29] H. Deng, M. P. Hertzberg, M. H. Namjoo, and A. Masoumi, Can light dark matter solve the core-cusp problem?, arXiv:1804.05921.

[30] C. J. Hogan and M. J. Rees, Axion miniclusters, Phys. Lett. B 205, 228 (1988).

[31] E. W. Kolb and I. I. Tkachev, Axion Miniclusters and Bose Stars, Phys. Rev. Lett. 71, 3051 (1993).
[32] J. Barranco and A. Bernal, Self-gravitating system made of axions, Phys. Rev. D 83, 043525 (2011).

[33] P. H. Chavanis and L. Delfini, Mass-radius relation of Newtonian self-gravitating Bose-Einstein condensates with short-range interactions, II: Numerical results, Phys. Rev. D 84, 043532 (2011).

[34] E. Braaten, A. Mohapatra, and H. Zhang, Dense Axion Stars, Phys. Rev. Lett. 117, 121801 (2016).

[35] E. D. Schiappacasse and M. P. Hertzberg, Analysis of dark matter axion clumps with spherical symmetry, J. Cosmol. Astropart. Phys. 01 (2018) 037; M. P. Hertzberg and E. D. Schiappacasse, Scalar dark matter clumps with angular momentum, arXiv:1804.07255.

[36] L. Visinelli, S. Baum, J. Redondo, K. Freese, and F. Wilczek, Dilute and dense axion stars, Phys. Lett. B 777, 64 (2018).

[37] E. Braaten, A. Mohapatra, and H. Zhang, Classical nonrelativistic effective field theories for a real scalar field, arXiv:1806.01898.

[38] H. Goldstein, C. Poole, and J. Safko, Classical Mechanics, 3rd ed. (Addison Wesley, Reading, MA, 2002), p. 373.

[39] Recently a paper appeared [40], which criticizes our approach to constructing nonrelativistic effective field theories. The authors raise a number of objections to the nonlocal field redefinition of our Eqs. (7), but they all appear to be based on misunderstandings. First, the authors say that it is enough to define a new field $\psi$ by our Eq. (6a), but that is not true. Equation (6a) cannot be inverted to define the new field $\psi$ in terms of the original relativistic field $\phi$. One way to see this is to notice that Eq. (6a) depends only on the real part of $e^{-i m t} \psi$, and therefore says nothing about the imaginary part. As a consequence, replacing $\psi$ by $\psi \rightarrow \psi^{\prime}=\psi+i e^{i m t} \rho(t, \mathbf{x})$, for any real-valued function $\rho(t, \mathbf{x})$, results in no change in $\phi(t, \mathbf{x})$. To fix $\psi(t, \mathbf{x})$ uniquely, at any given time, both of Eqs. (6a) and (6b) are needed. In this paper, however, we showed that there are advantages to using the nonlocal definition of Eqs. (7a) and (7b), although we showed in Appendix B that the local definition could alternatively be used. The arguments in Ref. [40] seem inconsistent with the fact that Eqs. (7a) and (7b) together allow a unique determination of $\psi(t, \mathbf{x})$ at any given time, in terms of $\phi(t, \mathbf{x})$ and $\pi(t, \mathbf{x})$, where $\psi(t, \mathbf{x})$ is given explicitly by our Eq. (9), which is an exact inverse to Eqs. (7a) and (7b). Using this one-to-one mapping of field configurations, replacing the two real functions $\phi(t, \mathbf{x})$ and $\pi(t, \mathbf{x})$ with one complex function $\psi(t, \mathbf{x})$, we construct a theory for $\psi(t, \mathbf{x})$ that is exactly equivalent to the original relativistic theory. After that, we make approximations that are appropriate in the nonrelativistic regime. The authors go on to argue that since our equation of motion is first order in the time derivative but infinite-order in spatial derivatives, it requires an infinite number of initial conditions. But our initial value problem is exactly equivalent to the standard case: given an initial value for the spatial function $\psi\left(t_{i}, \mathbf{x}\right)$ (which is equivalent to knowing $\phi\left(t_{i}, \mathbf{x}\right)$ and $\left.\pi\left(t_{i}, \mathbf{x}\right)\right)$, the differential equation determines the time derivative, so one can find the function at a slightly later time. Next the authors point out that in the free-field theory case $(\lambda=0)$, our equation of motion gives only a 
positive frequency solution for $\psi(t, \mathbf{x})$. They claim that therefore our treatment does not contain all the information of the complete theory. However, the negative frequency solutions are all contained in $\psi^{*}(t, \mathbf{x})$, and both $\psi(t, \mathbf{x})$ and $\psi^{*}(t, \mathbf{x})$ are used to reconstruct $\phi(t, \mathbf{x})$. Again, there is an exact one-to-one mapping, so any solution for $\phi(t, \mathbf{x})$ and $\pi(t, \mathbf{x})$ can be reconstructed by knowing $\psi(t, \mathbf{x})$, and vice versa. We also point out that our equation of motion for $\psi_{s}(t, \mathbf{x})$, through second order in small quantities, agrees exactly with Refs. [11] and [20].

[40] J. T. Firouzjaee, A. Rostami, and M. Bahmanabadi, Revisiting nonrelativistic limits of field theory: Antiparticles, arXiv: 1804.08600 . 